PREPARED FOR THE U.S. DEPARTMENT OF ENERGY, UNDER CONTRACT DE-AC02-76CH03073

PPPL-3789

PPPL-3789

UC-70

Kinetic Description of Intense Beam Propagation through a Periodic Focusing Field for Uniform Phase-space Density

by

Ronald C. Davidson, Hong Qin, Stephan I. Tzenov, and Edward A. Startsev

February 2003

NM|

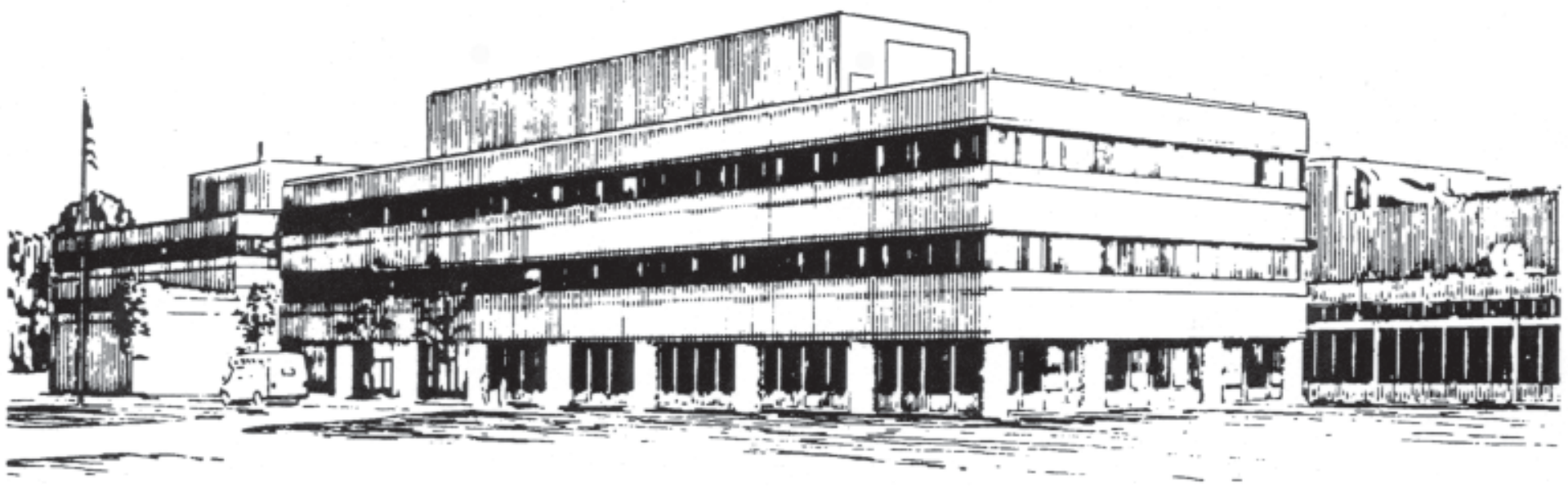

PRINCETON PLASMA PHYSICS LABORATORY PRINCETON UNIVERSITY, PRINCETON, NEW JERSEY 


\section{PPPL Reports Disclaimer}

This report was prepared as an account of work sponsored by an agency of the United States Government. Neither the United States Government nor any agency thereof, nor any of their employees, makes any warranty, express or implied, or assumes any legal liability or responsibility for the accuracy, completeness, or usefulness of any information, apparatus, product, or process disclosed, or represents that its use would not infringe privately owned rights. Reference herein to any specific commercial product, process, or service by trade name, trademark, manufacturer, or otherwise, does not necessarily constitute or imply its endorsement, recommendation, or favoring by the United States Government or any agency thereof. The views and opinions of authors expressed herein do not necessarily state or reflect those of the United States Government or any agency thereof.

\section{Availability}

This report is posted on the U.S. Department of Energy's Princeton Plasma Physics Laboratory Publications and Reports web site in Fiscal Year 2003. The home page for PPPL Reports and Publications is: http://www.pppl.gov/pub_report/

DOE and DOE Contractors can obtain copies of this report from:

U.S. Department of Energy

Office of Scientific and Technical Information

DOE Technical Information Services (DTIS)

P.O. Box 62

Oak Ridge, TN 37831

Telephone: (865) 576-8401

Fax: (865) 576-5728

Email: reports@adonis.osti.gov

This report is available to the general public from:

National Technical Information Service

U.S. Department of Commerce

5285 Port Royal Road

Springfield, VA 22161

Telephone: $1-800-553-6847$ or

(703) $605-6000$

Fax: (703) 321-8547

Internet: http://www.ntis.gov/ordering.htm 


\title{
Kinetic Description of Intense Beam Propagation Through a Periodic Focusing Field for Uniform Phase-Space Density
}

\author{
Ronald C. Davidson, Hong Qin, Stephan I. Tzenov, and Edward A. Startsev \\ Plasma Physics Laboratory, Princeton University, Princeton, NJ 08543
}

\begin{abstract}
The Vlasov-Maxwell equations are used to investigate the nonlinear evolution of an intense sheet beam with distribution function $f_{b}\left(x, x^{\prime}, s\right)$ propagating through a periodic focusing lattice $\kappa_{x}(s+S)=\kappa_{x}(s)$, where $S=$ const is the lattice period. The analysis considers the special class of distribution functions with uniform phase-space density $f_{b}\left(x, x^{\prime}, s\right)=A=$ const inside of the simply-connected boundary curves, $x_{+}^{\prime}(x, s)$ and $x_{-}^{\prime}(x, s)$, in the two-dimensional phase space $\left(x, x^{\prime}\right)$. Coupled nonlinear equations are derived describing the self-consistent evolution of the boundary curves, $x_{+}^{\prime}(x, s)$ and $x_{-}^{\prime}(x, s)$, and the self-field potential $\psi(x, s)=e_{b} \phi(x, s) / \gamma_{b} m_{b} \beta_{b}^{2} c^{2}$. The resulting model is shown to be exactly equivalent to a (truncated) warm-fluid description with zero heat flow and triple-adiabatic equation-of-state with scalar pressure $P_{b}(x, s)=$ const $\times\left[n_{b}(x, s)\right]^{3}$. Such a fluid model is amenable to direct analysis by transforming to Lagrangian variables following the motion of a fluid element. Specific examples of periodically-focused beam equilibria are presented, ranging from a finite-emittance beam in which the boundary curves in phase space $\left(x, x^{\prime}\right)$ correspond to a pulsating parallelogram, to a cold beam in which the number density of beam particles, $n_{b}(x, s)$, exhibits large-amplitude periodic oscillations. For the case of a sheet beam with uniform phase-space density, the present analysis clearly demonstrates the existence of periodically-focused beam equilibria without the undesirable feature of an inverted population in phase space that is characteristic of the Kapchinskij-Vladimirskij beam distribution.
\end{abstract}

PACS numbers: 29.27.Bd, 41.75.-i, 41.85.-p 


\section{INTRODUCTION}

Periodic focusing accelerators, transport systems and storage rings [1-5] have a wide range of applications ranging from basic research in high energy and nuclear physics, to applications such as heavy ion fusion, spallation neutron sources, tritium production, and nuclear waste transmutation. Of particular importance at the high beam currents and charge densities of practical interest are the effects of the intense self fields produced by the beam space charge and current on determining detailed equilibrium, stability and transport properties. In general, a complete description of collective processes in intense charged particle beams is provided by the nonlinear Vlasov-Maxwell equations [1] for the self-consistent evolution of the beam distribution function, $f_{b}(\mathbf{x}, \mathbf{p}, t)$, and the electric and magnetic fields, $\mathbf{E}(\mathbf{x}, t)$ and $\mathbf{B}(\mathbf{x}, t)$. While considerable progress has been made in analytical and numerical simulation studies of intense beam propagation [6-34], the effects of finite geometry and intense self fields often make it difficult to obtain detailed predictions of beam equilibrium, stability and transport properties based on the Vlasov-Maxwell equations. For example, the only known fully self-consistent equilibrium solution (including electric and magnetic self fields) to the nonlinear Vlasov-Maxwell equations for an intense beam propagating through a periodic focusing field configuration is the so-called Kapchinskij-Vladimirskij (KV) distribution function $f_{b}^{K V}[1,6-9]$. Such a distribution, due to its highly inverted population in phase space, of course is of very limited practical interest. While Hamiltonian averaging techniques have been developed [31-34] that justify the smooth-focusing approximation and thereby permit investigation of a whole class of (approximate) beam equilibria, these averaging techniques typically require sufficiently small vacuum phase advance $\left(\sigma_{v a c}<60^{\circ}\right.$, say $)$ and other approximations for their validity. Therefore, whether or not there exist periodically-focused non-KV solutions to the Vlasov-Maxwell equations remains a question of continued fundamental importance, which we examine in this paper for an intense sheet beam propagating through a periodic focusing field.

To briefly summarize, the present analysis considers an intense sheet beam which is infinite in the $y$-dimension and propagates in the $z$-direction with average axial velocity $V_{b}=\beta_{b} c=$ const and directed kinetic energy $\left(\gamma_{b}-1\right) m_{b} c^{2}$, where $\gamma_{b}=\left(1-\beta_{b}^{2}\right)^{-1 / 2}$ is the relativistic mass factor, $m_{b}$ is the rest mass of a beam particle, and $c$ is the speed of light in vacuo. The beam propagates through a periodic focusing lattice $\kappa_{x}(s+S)=\kappa_{x}(s)$, where 
$S=$ const is the lattice period, which provides transverse confinement of the beam particles in the $x$-direction. The self-consistent evolution of the system is described by the nonlinear Vlasov-Poisson equations [Eqs. (1) and (2)] for the beam distribution function, $f_{b}\left(x, x^{\prime}, s\right)$, and the normalized self-field potential, $\psi(x, s)=e_{b} \phi(x, s) / \gamma_{b} m_{b} \beta_{b}^{2} c^{2}$. Here $x^{\prime}=d x / d s$ is the dimensionless velocity, $e_{b}$ is the charge of a beam particle, and $\phi(x, s)$ is the space-charge potential. In the present analysis, we consider the special class of distribution functions with uniform phase-space density in which $f_{b}\left(x, x^{\prime}, s\right)=A=$ const [Eq. (23)] inside of the simplyconnected boundaries, $x_{+}^{\prime}(x, s)$ and $x_{-}^{\prime}(x, s)$, in the phase space $\left(x, x^{\prime}\right)$. Coupled equations are derived describing the self-consistent evolution of the boundary curves, $x_{+}^{\prime}(x, s)$ and $x_{-}^{\prime}(x, s)$, and the self-field potential $\psi(x, s)$ [Eqs. (27)-(29)]. Quite remarkably, the resulting model is found to be exactly equivalent to a (truncated) warm-fluid description with zero heat flow and triple-adiabatic equation-of-state [Eqs. (33), (35), (36) and (37)]. Such a fluid model is amenable to direct analysis by transforming to Lagrangian variables following the motion of a fluid element [35, 36].

The organization of the paper is the following. The theoretical model based on the Vlasov-Maxwell equations is summarized in Sec. II, including a derivation of the statistical rate equations describing the general nonlinear evolution of the rms beam thickness, centroid motion, and the unnormalized beam emittance. In Sec. III, the Vlasov-Maxwell equations are simplified for the case of a sheet beam with uniform phase space density; the dynamical equations are derived for the boundary curves, $x_{+}^{\prime}(x, s)$ and $x_{-}^{\prime}(x, s)$, in phase space (Sec. III.A); and the equivalence of the model to a (truncated) warm-fluid description with triple-adiabatic equation-of-state is demonstrated (Sec. III.B). For the specific example of a pulsating parallelogram with uniform phase space density, closed dynamical equations for the self-consistent evolution of the system are derived in Sec. IV. Finally, in Sec. V, the (closed) warm-fluid model derived in Sec. III is transformed to Lagrangian variables $\left(x_{0}, \tau\right)$ following the motion of a fluid element. This leads to a single nonlinear partial differential equation [Eq. (76)] for the number density $n_{b}\left(x_{0}, \tau\right)$ of beam particles. Specific numerical examples corresponding to large-amplitude collective oscillations in the cold-beam limit are also considered in Sec. V, including back transformation to the laboratory frame.

For the case of a sheet beam with uniform phase-space density, the present analysis clearly demonstrates the existence of periodically focused beam equilibria without the undesirable feature of an inverted population in phase space that is characteristic of the 
Kapchinskij-Vladimirskij beam distribution. It should be emphasized that the existence of periodically-focused beam equilibrium for a non-KV distribution with uniform density in the two-dimensional phase space $\left(x, x^{\prime}\right)$ does not imply that periodically focused beam equilibria exist for non-KV beam distributions in four- and six-dimensions.

\section{THEORETICAL MODEL}

In the present analysis, we consider an intense sheet beam, made up of particles with charge $e_{b}$ and rest mass $m_{b}$, which is infinite in the $y$-dimension $(\partial / \partial y=0)$, and propagates in the $z$-direction with average axial velocity $V_{b}=\beta_{b} c$ and directed kinetic energy $\left(\gamma_{b}-1\right) m_{b} c^{2}$, where $\gamma_{b}=\left(1-\beta_{b}^{2}\right)^{-1 / 2}$ is the relativistic mass factor, and $c$ is the speed of light in vacuo. The beam propagates through a periodic focusing lattice $\kappa_{x}(s+S)=\kappa_{x}(s)$, where $S=$ const. is the lattice period, which provides transverse confinement of the beam particles in the $x$-direction. Introducing the (dimensionless) selffield potential $\psi(x, s) \equiv e_{b} \phi(x, s) / \gamma_{b} m_{b} \beta_{b}^{2} c^{2}$, where $\phi(x, s)$ is the electrostatic (space-charge) potential, the nonlinear Vlasov-Maxwell equations describing the self-consistent nonlinear beam dynamics and collective processes in the paraxial approximation are given by [37]

$$
\begin{gathered}
\frac{\partial f_{b}}{\partial s}+x^{\prime} \frac{\partial f_{b}}{\partial x}-\left(\kappa_{x}(s) x+\frac{\partial \psi}{\partial x}\right) \frac{\partial f_{b}}{\partial x^{\prime}}=0, \\
\frac{\partial^{2} \psi}{\partial x^{2}}=-\frac{2 \pi K_{b}}{N_{b}} \int d x^{\prime} f_{b} .
\end{gathered}
$$

In Eqs. (1) and (2), $f_{b}\left(x, x^{\prime}, s\right)$ is the distribution of particles in the two-dimensional phase space $\left(x, x^{\prime}\right), x^{\prime}=d x / d s$ is the (dimensionless) velocity in the $x$-direction, $s=s_{0}+\beta_{b} c t$ is a normalized time variable, the focusing coefficient $\kappa_{x}(s+S)=\kappa_{x}(s)$ has dimensions (length) ${ }^{-2}$, and

$$
n_{b}(x, s)=\int d x^{\prime} f_{b}\left(x, x^{\prime}, s\right),
$$

is the number density of beam particles (number of beam particles per unit volume). For

present purposes, we assume that perfectly conducting walls are located at $x= \pm x_{w}$ (the case where $x_{w} \rightarrow \infty$ is not excluded), and enforce the boundary conditions

$$
\psi\left(x= \pm x_{w}, s\right)=\text { const } .
$$


in solving Eqs. (1) and (2). Furthermore, the constants $K_{b}$ and $N_{b}$ occurring in Eqs. (1) and (2) are defined by

$$
K_{b}=\frac{2 N_{b} e_{b}^{2}}{\gamma_{b}^{3} m_{b} \beta_{b}^{2} c^{2}}=\text { const. }
$$

and

$$
N_{b}=\int d x d x^{\prime} f_{b}\left(x, x^{\prime}, s\right)=\text { const }
$$

Here, $K_{b}$ is the normalized self-field perveance, with dimensions of (length) ${ }^{-1}$, and $N_{b}=$ $\int d x n_{b}(x, s)$ is the area number density of beam particles, with dimensions of (length) ${ }^{-2}$. The validity of Eqs. (1) and (2) assumes negligibly small axial momentum spread in the $z$-direction, and that the particle motions are nonrelativistic in a frame of reference moving with the beam (axial velocity in the $z$-direction $=\beta_{b} c=$ const.). We further assume that

$$
\begin{gathered}
f\left(x, x^{\prime}= \pm \infty, s\right)=0, \\
f\left(|x|>\left|x_{0}\right|, x^{\prime}, s\right)=0,
\end{gathered}
$$

such that there are no beam particles beyond some transverse $x$-dimension $|x|=\left|x_{0}\right|<x_{w}$.

The Vlasov-Maxwell equations (1) and (2), subject to the boundary conditions in Eqs. (4) and (7), are valid for an intense sheet beam in the paraxial approximation, and can be used to describe the nonlinear beam dynamics and collective processes in the phase space $\left(x, x^{\prime}\right)$ over a wide range of system parameters and applied focusing field configurations $\kappa_{x}(s+S)=\kappa_{x}(s)$. While the full solutions for $f_{b}\left(x, x^{\prime}, s\right)$ and $\psi(x, s)$ are of special interest, it is also possible to derive exact equations for the evolution of statistical averages $\langle\chi\rangle(s)$. Here, the statistical average of a general phase function $\chi\left(x, x^{\prime}, s\right)$ over the phase-space distribution $f_{b}\left(x, x^{\prime}, s\right)$ is defined by

$$
\langle\chi\rangle=\frac{1}{N_{b}} \int d x d x^{\prime} \chi f_{b}
$$

where $N_{b}=\int d x n_{b}=\int d x d x^{\prime} f_{b}=$ const. is the area number density of beam particles in the sheet beam. For $\chi=x$ and $\chi=x^{\prime}$, taking the appropriate moments of Eq. (1), readily gives [38]

$$
\frac{d}{d s}\langle x\rangle=\left\langle x^{\prime}\right\rangle
$$

and

$$
\frac{d}{d s}\left\langle x^{\prime}\right\rangle+\kappa_{x}(s)\langle x\rangle=-\left\langle\frac{\partial \psi}{\partial x}\right\rangle
$$


where use has been made of Eq. (7). Substituting Eq. (9) into Eq. (10) readily gives for the evolution of the centroid location $\langle x\rangle(s)$,

$$
\frac{d^{2}}{d s^{2}}\langle x\rangle+\kappa_{x}(s)\langle x\rangle=-\left\langle\frac{\partial \psi}{\partial x}\right\rangle .
$$

In Eq. (11), the average self-field force, $-\langle\partial \psi / \partial x\rangle$, is determined self-consistently from Eqs. (1), (2) and (8). Similarly, it can be shown (exactly) from Eqs. (1) and (8) that

$$
\frac{d}{d s}\left\langle x^{2}\right\rangle=2\left\langle x x^{\prime}\right\rangle
$$

and

$$
\frac{d}{d s}\left\langle x x^{\prime}\right\rangle+\kappa_{x}(s)\left\langle x^{2}\right\rangle=\left\langle x^{\prime 2}\right\rangle-\left\langle x \frac{\partial \psi}{\partial x}\right\rangle,
$$

or equivalently,

$$
\frac{d^{2}}{d s^{2}} \frac{1}{2}\left\langle x^{2}\right\rangle+\kappa_{x}(s)\left\langle x^{2}\right\rangle=\left\langle x^{\prime 2}\right\rangle-\left\langle x \frac{\partial \psi}{\partial x}\right\rangle .
$$

In addition, it can be shown from Eqs. (1) and (8) that

$$
\frac{d}{d s}\left\langle x^{\prime 2}\right\rangle+2 \kappa_{x}(s)\left\langle x x^{\prime}\right\rangle=-2\left\langle x^{\prime} \frac{\partial \psi}{\partial x}\right\rangle,
$$

or equivalently,

$$
\frac{d}{d s}\left\langle x^{\prime 2}\right\rangle+\kappa_{x}(s) \frac{d}{d s}\left\langle x^{2}\right\rangle=-2\left\langle x^{\prime} \frac{\partial \psi}{\partial x}\right\rangle,
$$

where use is made of Eq. (12). Equations (11), (14) and (16), derived from the Vlasov equation (1), are exact equations describing the nonlinear evolution of the statistical averages $\langle x\rangle(s),\left\langle x^{2}\right\rangle(s)$ and $\left\langle x^{\prime 2}\right\rangle(s)$. In a similar manner, making use of Eq. (1), dynamical equations can be derived for the evolution of statistical averages for higher-order moments, $\left\langle x^{m}\right\rangle,\left\langle x^{\prime n}\right\rangle$ and $\left\langle x^{p} x^{q}\right\rangle$, for $m>2, n>2$, etc.

For future reference, it is convenient to rewrite Eq. (14) in terms of the mean-square beam dimension $X_{b}^{2}(s)$ defined by

$$
X_{b}^{2}=\left\langle(x-\langle x\rangle)^{2}\right\rangle
$$

We further make use of the identities

$$
\begin{aligned}
& \left\langle(x-\langle x\rangle)^{2}\right\rangle=\left\langle x^{2}\right\rangle-\langle x\rangle^{2}, \\
& \left\langle\left(x^{\prime}-\left\langle x^{\prime}\right\rangle\right)^{2}\right\rangle=\left\langle x^{\prime 2}\right\rangle-\left\langle x^{\prime}\right\rangle^{2}, \\
& \left\langle(x-\langle x\rangle)\left(x^{\prime}-\left\langle x^{\prime}\right\rangle\right)\right\rangle=\frac{1}{2} \frac{d}{d s}\left\langle(x-\langle x\rangle)^{2}\right\rangle .
\end{aligned}
$$


Substituting Eqs. (17) and (18) into Eq. (14), and making use of Eq. (11) to eliminate $\left(d^{2} / d s^{2}\right)\langle x\rangle$, we readily obtain

$$
\frac{d^{2}}{d s^{2}} \frac{1}{2} X_{b}^{2}+\kappa_{x}(s) X_{b}^{2}=\left\langle\left(x^{\prime}-\left\langle x^{\prime}\right\rangle\right)^{2}\right\rangle-\left\langle(x-\langle x\rangle) \frac{\partial \psi}{\partial x}\right\rangle .
$$

Subtracting out the centroid motion, it is convenient to introduce the unnormalized beam emittance $\epsilon_{x}(s)$ defined by

$$
\begin{aligned}
\frac{1}{4} \epsilon_{x}^{2}(s) & =\left\langle\left(x^{\prime}-\left\langle x^{\prime}\right\rangle\right)^{2}\right\rangle\left\langle(x-\langle x\rangle)^{2}\right\rangle-\left\langle(x-\langle x\rangle)\left(x^{\prime}-\left\langle x^{\prime}\right\rangle\right)\right\rangle^{2} \\
& =\left\langle\left(x^{\prime}-\left\langle x^{\prime}\right\rangle\right)^{2}\right\rangle X_{b}^{2}-X_{b}^{2}\left(\frac{d X_{b}}{d s}\right)^{2},
\end{aligned}
$$

where $X_{b}^{2}=\left\langle(x-\langle x\rangle)^{2}\right\rangle$, and use has been made of Eq. (18). Using Eq. (20) to eliminate $\left\langle\left(x^{\prime}-\left\langle x^{\prime}\right\rangle\right)^{2}\right\rangle$ in favor of $\epsilon_{x}^{2}(s)$ in Eq. (19) gives directly the dynamical equation

$$
\frac{d^{2} X_{b}}{d s^{2}}+\kappa_{x}(s) X_{b}=\frac{\epsilon_{x}^{2}(s)}{4 X_{b}^{3}}-\frac{1}{X_{b}}\left\langle(x-\langle x\rangle) \frac{\partial \psi}{\partial x}\right\rangle .
$$

Equation (21) is fully equivalent to Eq. (14), and describes the nonlinear evolution of the rms beam thickness $X_{b}(s)$.

In a similar manner, Eq. (16) for $\left\langle x^{\prime 2}\right\rangle(s)$ can be replaced by a dynamical equation for the unnormalized beam emittance. Without presenting algebraic details, making use of Eqs. (11), (14), (16) and (18), we obtain

$$
\begin{aligned}
\frac{d}{d s} \frac{1}{8} \epsilon_{x}^{2}(s) & =-\left\langle(x-\langle x\rangle)^{2}\right\rangle\left\langle\left(x^{\prime}-\left\langle x^{\prime}\right\rangle\right) \frac{\partial \psi}{\partial x}\right\rangle \\
& +\left\langle(x-\langle x\rangle)\left(x^{\prime}-\left\langle x^{\prime}\right\rangle\right)\right\rangle\left\langle(x-\langle x\rangle) \frac{\partial \psi}{\partial x}\right\rangle \\
& =-X_{b}^{2}\left\langle\left(x^{\prime}-\left\langle x^{\prime}\right\rangle\right) \frac{\partial \psi}{\partial x}\right\rangle+X_{b} \frac{d X_{b}}{d s}\left\langle(x-\langle x\rangle) \frac{\partial \psi}{\partial x}\right\rangle .
\end{aligned}
$$

Equation (22) shows clearly that space-charge effects (proportional to $\partial \psi / \partial x$ ) generally cause a variation in the beam emittance $\epsilon_{x}(s)$. Only in the limit of very low beam intensity $(|\partial \psi / \partial x| \rightarrow 0)$, or very special choices of distribution function $f_{b}\left(x, x^{\prime}, s\right)$ (see Sec. IV), is the emittance a conserved quantity.

To summarize, Eqs. (11), (21) and (22) are exact consequences of the Vlasov equation (1) and describe the self-consistent dynamical evolution of the beam centroid $\langle x\rangle(s)$, rms thickness $X_{b}(s)$, and emittance $\epsilon_{x}(s)$. In general, Eqs. (11), (21) and (22) are not closed 
dynamical equations because the statistical averages $\langle\partial \psi / \partial x\rangle,\langle(x-\langle x\rangle) \partial \psi / \partial x\rangle$, etc., require a knowledge of the self-field potential $\psi(x, s)$ which is determined self-consistently in terms of the distribution function $f_{b}\left(x, x^{\prime}, s\right)$ from the Vlasov-Maxwell equations (1) and (2).

\section{NONLINEAR VLASOV-MAXWELL EQUATIONS FOR UNIFORM PHASE- SPACE DENSITY}

We now return to the Vlasov-Maxwell equations (1) and (2) for the distribution function $f_{b}\left(x, x^{\prime}, s\right)$ and self-field potential $\psi(x, s)$. For specified applied focusing field $\kappa_{x}(s+S)=$ $\kappa_{x}(s)$ and initial distribution function $f_{b}\left(x, x^{\prime}, s=0\right)$, obtaining the solutions to Eqs. (1) and (2) is generally difficult analytically, although solutions to Eqs. (1) and (2) are accessible using nonlinear $\delta f$ simulation techniques[22-25]. For present purposes, we consider a special case where considerable analytical simplification occurs in the analysis of Eqs. (1) and (2). In particular, as illustrated in Fig. 1, we consider the case where the distribution function $f_{b}\left(x, x^{\prime}, s\right)$ has constant phase-space density (independent of $x, x^{\prime}$ and $s$ ) within the simplyconnected boundary curves $x_{+}^{\prime}(x, s)$ and $x_{-}^{\prime}(x, s)$, and zero phase-space density outside.

That is, we take

$$
f_{b}\left(x, x^{\prime}, s\right)= \begin{cases}A=\text { const. }, & x_{-}^{\prime}(x, s)<x^{\prime}<x_{+}^{\prime}(x, s), \\ 0, & \text { otherwise } .\end{cases}
$$

If $f_{b}\left(x, x^{\prime}, s\right)$ satisfies Eq. (23) initially at $s=0$, then the nonlinear Vlasov equation (1) assures that the phase-space density remains constant at subsequent values of $s$ as the boundary curves $x_{+}^{\prime}(x, s)$ and $x_{-}^{\prime}(x, s)$ distort and evolve nonlinearly in response to the applied focusing field and the self-generated fields. Of course as the system evolves, Eq. (1) assures that

$$
d N_{b} / d s=(d / d s) \int d x d x^{\prime} f_{b}\left(x, x^{\prime}, s\right)=0,
$$

or equivalently from Eq. (23) and Fig. 1,

$$
N_{b}=A \int_{x_{b}^{-}(s)}^{x_{b}^{+}(s)} d x\left[x_{+}^{\prime}(x, s)-x_{-}^{\prime}(x, s)\right]=\text { const. }
$$

That is, no matter how complicated the evolution of the boundary curves, $x_{+}^{\prime}(x, s)$ and $x_{-}^{\prime}(x, s)$ in Fig. 1, the total area within the phase-space boundary remains constant. In the subsequent analysis, we assume that the boundary curves, $x_{+}^{\prime}(x, s)$ and $x_{-}^{\prime}(x, s)$, in Fig. 1 remain single-valued functions of $x$ as the system evolves. 


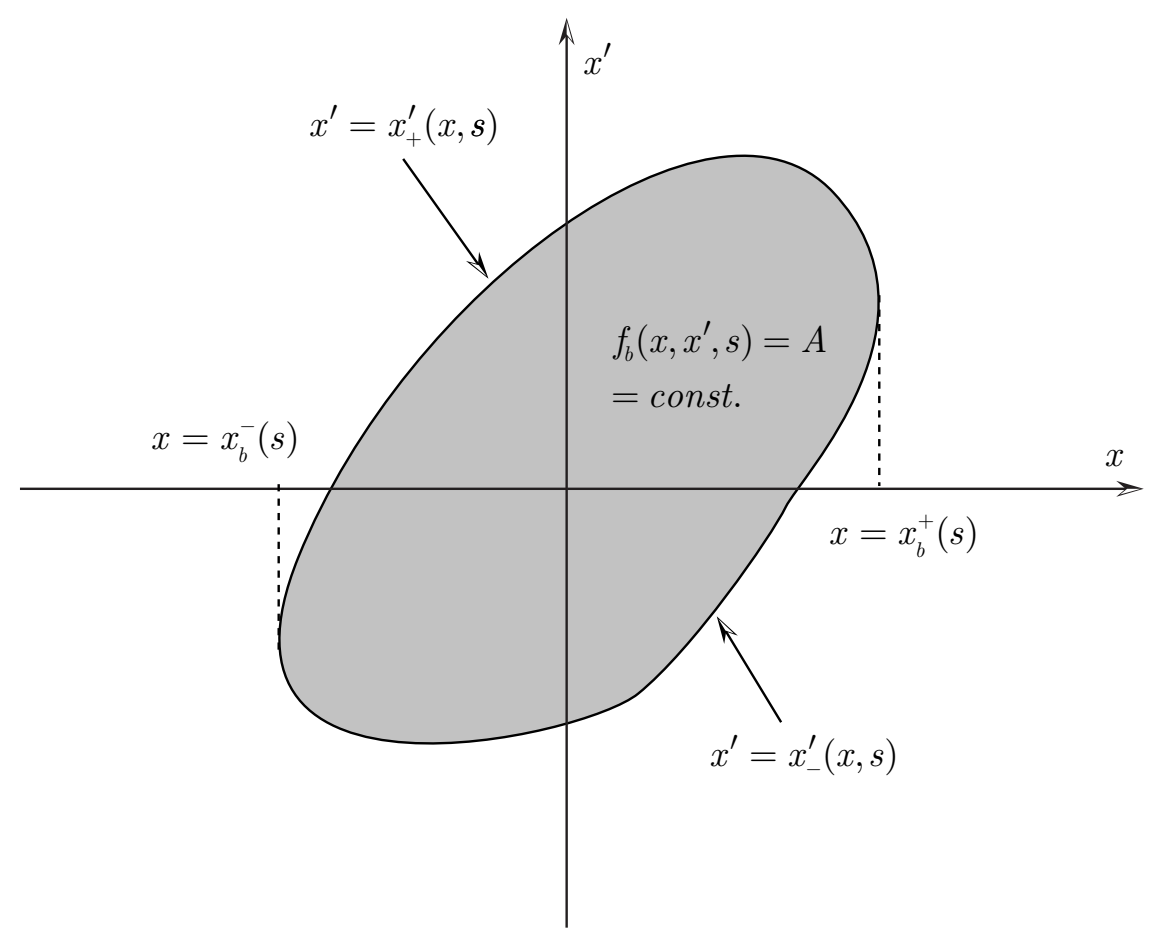

FIG. 1: Phase-space plot of distribution function with uniform phase-space density $f_{b}\left(x, x^{\prime}, s\right)=$ $A=$ const. in the region $x_{-}^{\prime}(x, s)<x^{\prime}<x_{+}^{\prime}(x, s)$ and $x_{b}^{-}(s)<x<x_{b}^{+}(s)$ [Eq. (23)].

\section{A. Dynamical Equations for $x_{+}^{\prime}(x, s)$ and $x_{-}^{\prime}(x, s)$}

We now make use of Eqs. (1) and (23) to derive exact dynamical equations for the boundary curves $x_{+}^{\prime}(x, s)$ and $x_{-}^{\prime}(x, s)$. Referring to Fig. 1 and Eq. (23), we operate on Eq. (1) with $\int_{-\infty}^{\infty} d x^{\prime} \cdots$. This readily gives

$$
\frac{\partial}{\partial s}\left(x_{+}^{\prime}-x_{-}^{\prime}\right)+\frac{\partial}{\partial x} \frac{1}{2}\left(x_{+}^{\prime 2}-x_{-}^{\prime 2}\right)=0
$$

for $x_{b}^{-}(s)<x<x_{b}^{+}(s)$. Here, use has been made of $\int_{-\infty}^{\infty} d x^{\prime} \partial f_{b} / \partial x^{\prime}=0, \int_{-\infty}^{\infty} d x^{\prime} f_{b}=$ $A\left(x_{+}^{\prime}-x_{-}^{\prime}\right)$, and $\int_{-\infty}^{\infty} d x^{\prime} x^{\prime} f_{b}=A(1 / 2)\left(x_{+}^{\prime 2}-x_{-}^{\prime 2}\right)$. In a similar manner, operating on Eq. (1) with $\int_{-\infty}^{\infty} d x^{\prime} x^{\prime} \cdots$, and making use of $\int_{-\infty}^{\infty} d x^{\prime} x^{\prime} \partial f_{b} / \partial x^{\prime}=-\int_{-\infty}^{\infty} d x^{\prime} f_{b}$, we obtain

$$
\frac{\partial}{\partial s} \frac{1}{2}\left(x_{+}^{\prime 2}-x_{-}^{\prime 2}\right)+\frac{\partial}{\partial x} \frac{1}{3}\left(x_{+}^{\prime 3}-x_{-}^{\prime 3}\right)=-\left(x_{+}^{\prime}-x_{-}^{\prime}\right)\left(\kappa_{x}(s) x+\frac{\partial \psi}{\partial x}\right),
$$

for $x_{b}^{-}(s)<x<x_{b}^{+}(s)$. Finally, making use of $\int d x^{\prime} f_{b}=A\left(x_{+}^{\prime}-x_{-}^{\prime}\right)$ for the choice of distribution function in Eq. (23), it is straightforward to show that Eq. (2) for the self-field 
potential $\psi(x, s)$ reduces to

$$
\frac{\partial^{2} \psi}{\partial x^{2}}= \begin{cases}0, & -x_{w} \leq x<x_{b}^{-}(s), \\ -\frac{2 \pi K_{b}}{N_{b}} A\left(x_{+}^{\prime}-x_{-}^{\prime}\right), & x_{b}^{-}(s)<x<x_{b}^{+}(s), \\ 0, & x_{b}^{+}(s)<x \leq x_{w} .\end{cases}
$$

Equations (25) and (26) can be combined to give separate dynamical equations for $x_{+}^{\prime}(x, s)$ and $x_{-}^{\prime}(x, s)$. Some straightforward algebraic manipulation gives

$$
\begin{gathered}
\frac{\partial}{\partial s} x_{+}^{\prime}+x_{+}^{\prime} \frac{\partial}{\partial x} x_{+}^{\prime}=-\kappa_{x}(s) x-\frac{\partial \psi}{\partial x}, \\
\frac{\partial}{\partial s} x_{-}^{\prime}+x_{-}^{\prime} \frac{\partial}{\partial x} x_{-}^{\prime}=-\kappa_{x}(s) x-\frac{\partial \psi}{\partial x},
\end{gathered}
$$

for $x_{b}^{-}(s)<x<x_{b}^{+}(s)$. Equations (28) and (29) are exactly equivalent to Eqs. (25) and (26). Note that Eqs. (28) and (29) are simply statements that the acceleration of the upper $\left(x_{+}^{\prime}\right)$ and lower $\left(x_{-}^{\prime}\right)$ phase-space boundaries in Fig. 1 is equal to $-\kappa_{x} x-\partial \psi / \partial x$, which corresponds to the combined effects of the applied focusing force $\left(-\kappa_{x} x\right)$ and the self-field force $(-\partial \psi / \partial x)$.

In summary, for the case of constant phase-space density in Eq. (23) and Fig. 1, there has been an enormous simplification in the kinetic description based on the Vlasov-Maxwell equations (1) and (2). In particular, Eqs. (1) and (2), which are partial differential equations in the three variables $\left(x, x^{\prime}, s\right)$, are replaced exactly by Eqs. (27)-(29), which are partial differential equations in the two variables $(x, s)$. Equations (27)-(29) can be solved analytically in at least one case of special interest (Sec. IV), and can be integrated numerically for a wide range of initial phase-space boundaries, $x_{+}^{\prime}(x, s=0)$ and $x_{-}^{\prime}(x, s=0)$.

\section{B. Equivalence to a Warm-Fluid Model}

It is instructive to re-cast the basic equations derived in Sec. III.A in a form familiar in macroscopic warm-fluid descriptions widely used in plasma physics[35]. In this regard, we introduce the number density $n_{b}(x, s)$, and the (normalized) macroscopic flow velocity, 
$V_{x b}(x, s)$, pressure, $P_{b}(x, s)$, and heat flow, $Q_{b}(x, s)$, defined by

$$
\begin{aligned}
n_{b} & =\int d x^{\prime} f_{b}, \\
n_{b} V_{x b} & =\int d x^{\prime} x^{\prime} f_{b}, \\
P_{b} & =\int d x^{\prime}\left(x^{\prime}-V_{x b}\right)^{2} f_{b}, \\
Q_{b} & =\int d x^{\prime}\left(x^{\prime}-V_{x b}\right)^{3} f_{b} .
\end{aligned}
$$

Referring to Eq. (23) and Fig. 1, some straightforward algebra gives

$$
\begin{aligned}
n_{b} & =A\left(x_{+}^{\prime}-x_{-}^{\prime}\right), \\
n_{b} V_{x b} & =\frac{1}{2} A\left(x_{+}^{\prime 2}-x_{-}^{\prime 2}\right), \\
P_{b} & =\frac{1}{12 A^{2}}\left[A\left(x_{+}^{\prime}-x_{-}^{\prime}\right)\right]^{3}, \\
Q_{b} & =0
\end{aligned}
$$

for $x_{b}^{-}(s)<x<x_{b}^{+}(s)$. Note from Eq. (31) that the transverse flow velocity $V_{x b}(x, s)$ is given by

$$
V_{x b}=\frac{1}{2}\left(x_{+}^{\prime}+x_{-}^{\prime}\right)
$$

and the pressure $P_{b}(x, s)$ can be expressed as

$$
P_{b}=\frac{\hat{P}_{b 0}}{\hat{n}_{b 0}^{3}} n_{b}^{3},
$$

where $\hat{P}_{b 0} / \hat{n}_{b 0}^{3} \equiv 1 / 12 A^{2}=$ const. is a constant coefficient. Furthermore, because there is no 'skew' in the $x^{\prime}$-dependence of $f_{b}\left(x, x^{\prime}, s\right)$ in Eq. (23), the heat flow $Q_{b}$ is identically zero $\left(Q_{b}=0\right)$. Making use of the expressions for $n_{b} V_{x b}, P_{b}$ and $V_{x b}$ in Eqs. (31) and (32) gives the useful identity

$$
n_{b} V_{x b}^{2}+P_{b}=\frac{1}{3}\left(x_{+}^{\prime 3}-x_{-}^{\prime 3}\right)
$$

We now return to the basic dynamical equations for $x_{+}^{\prime}$ and $x_{-}^{\prime}$ derived in Eqs. (25) and (26) in Sec. III.A. Substituting Eqs. (31)-(34) into Eqs. (25) and (26) gives directly the familiar macroscopic continuity and force balance equations,

$$
\frac{\partial}{\partial s} n_{b}+\frac{\partial}{\partial x}\left(n_{b} V_{x b}\right)=0
$$


and

$$
\frac{\partial}{\partial s} n_{b} V_{x b}+\frac{\partial}{\partial x}\left(n_{b} V_{x b} V_{x b}\right)+\frac{\partial P_{b}}{\partial x}=-n_{b}\left(\kappa_{x}(s) x+\frac{\partial \psi}{\partial x}\right),
$$

where $P_{b}(x, s)=\left(\hat{P}_{b 0} / \hat{n}_{b 0}^{3}\right) n_{b}^{3}(x, s)$ satisfies the triple-adiabatic pressure relation in Eq. (33). Furthermore, Poisson's equation (27) is simply expressed as

$$
\frac{\partial^{2} \psi}{\partial x^{2}}=-\frac{2 \pi K_{b}}{N_{b}} n_{b}
$$

Finally, Eq. (36) can be further simplified by making use of Eqs. (33) and (35) to eliminate $\partial n_{b} / \partial s$. We readily obtain

$$
\frac{\partial}{\partial s} V_{x b}+V_{x b} \frac{\partial}{\partial x} V_{x b}+\frac{3}{2} \frac{\hat{P}_{b 0}}{\hat{n}_{b 0}^{3}} \frac{\partial}{\partial x} n_{b}^{2}=-\kappa_{x}(s) x-\frac{\partial \psi}{\partial x}
$$

in the region where $n_{b} \neq 0$. Equation (38) shows clearly that the transverse acceleration of a beam fluid element is produced by the combined effects of the pressure-gradient force $\left(-n_{b}^{-1} \partial P_{b} / \partial x\right)$, the applied focusing force $\left(-\kappa_{x} x\right)$, and the self-field force $(-\partial \psi / \partial x)$.

To summarize, for the case of constant phase-space density in Eq. (23) and Fig. 1, the macroscopic fluid description provided by Eqs. (33), (35), (36) and (37), or equivalently, Eqs. (35), (37) and (38), is fully equivalent to the nonlinear Vlasov-Maxwell equations (1) and (2). This remarkable simplification, i.e., closure of the macroscopic fluid equations with the first two velocity moments for $n_{b}(x, s)$ and $V_{x b}(x, s)$, is a consequence of the fact that the heat flow satisfies $Q_{b}(x, s)=0$ exactly for the class of beam distribution functions in Eq. (23) and Fig. 1 [35]. Similar to Sec. III.A, the Vlasov-Maxwell equations (1) and (2), which are nonlinear partial differential equations in the three variables $\left(x, x^{\prime}, s\right)$, have been replaced by the macroscopic fluid-Maxwell equations (35), (37) and (38), which are nonlinear partial differential equations in the two variables $(x, s)$. It should be pointed out that Eqs. (35), (37) and (38) are readily amenable to numerical solution, and can also be investigated analytically, e.g., by introducing a Lagrangian transformation to a frame of reference moving with velocity $V_{x b}(x, s)$ of a beam fluid element. Equations (35), (37) and (38) can be used to investigate detailed beam propagation properties over a wide range of focusing field configurations $\kappa_{x}(s+S)=\kappa_{x}(s)$ and system parameters. In this regard, it should be noted that the dynamical equations for the beam centroid $\langle x\rangle(s)$ [Eq. (11)], the rms beam thickness $X_{b}(s)$ [Eq. (21)], and the unnormalized beam emittance $\epsilon_{x}(s)$ [Eq. (22)], derived in Sec. II, also apply to the class of distribution functions described by Eq. (23) and Fig. 1. 


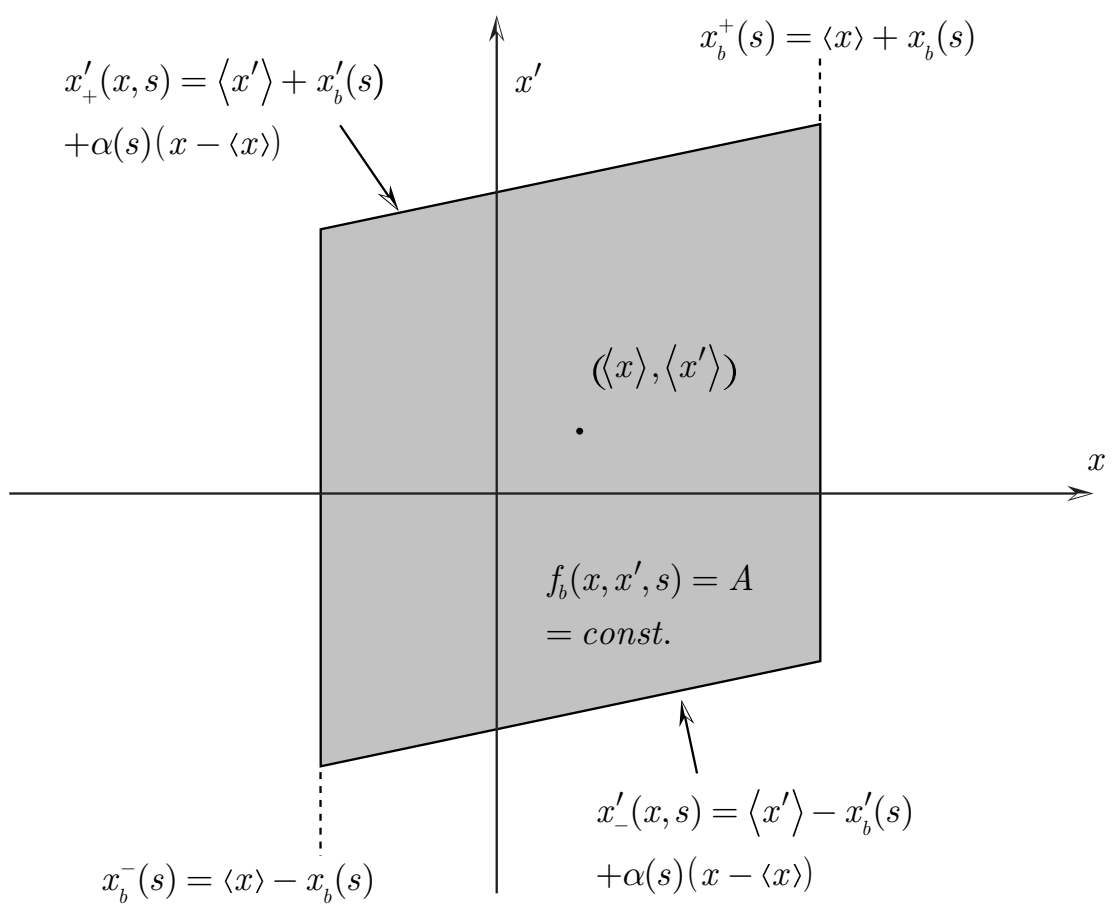

FIG. 2: Phase-space plot of distribution function $f_{b}\left(x, s^{\prime}, s\right)=A=$ const. in Eq. (40). Note that the boundary curves in the figure correspond to the parallelogram $x^{\prime}=x_{ \pm}^{\prime}(x, s)=\left\langle x^{\prime}\right\rangle \pm$ $x_{b}^{\prime}(s)+\alpha(s)(x-\langle x\rangle)$ and $x=x_{b}^{ \pm}(s)=\langle x\rangle \pm x_{b}(s)$, and that the parallelogram is centered at $\left(x, x^{\prime}\right)=\left(\langle x\rangle,\left\langle x^{\prime}\right\rangle\right)$.

\section{PULSATING PARALLELOGRAM WITH UNIFORM PHASE-SPACE DENSITY}

As an application of the analysis in Sec. III, we now consider the uniform phase-space distribution illustrated in Fig. 2, where the boundary curves $x_{+}^{\prime}(x, s), x_{-}^{\prime}(x, s), x_{b}^{+}(s), x_{b}^{-}(s)$ corresponds instantaneously to a parallelogram centered at the phase-space point $\left(\langle x\rangle,\left\langle x^{\prime}\right\rangle\right)$, where $\langle x\rangle(s)$ is the centroid position, and $\left\langle x^{\prime}\right\rangle(s)=(d / d s)\langle x\rangle(s)$ is the centroid velocity. Here, the boundary curves are defined by

$$
\begin{aligned}
x_{+}^{\prime}(x, s) & =\left\langle x^{\prime}\right\rangle(s)+x_{b}^{\prime}(s)+\alpha(s)[x-\langle x\rangle(s)], \\
x_{-}^{\prime}(x, s) & =\left\langle x^{\prime}\right\rangle(s)-x_{b}^{\prime}(s)+\alpha(s)[x-\langle x\rangle(s)], \\
x_{b}^{+}(s) & =\langle x\rangle(s)+x_{b}(s), \\
x_{b}^{-}(s) & =\langle x\rangle(s)-x_{b}(s),
\end{aligned}
$$


where $\alpha(s)$ is a (yet unspecified) s-dependent coefficient. The distribution function $f_{b}\left(x, x^{\prime}, s\right)$ with constant phase-space density consistent with Eq. (39) and Fig. 2 is given by

$$
f_{b}\left(x, x^{\prime}, s\right)= \begin{cases}A=\text { const. }, & \left\langle x^{\prime}\right\rangle-x_{b}^{\prime}+\alpha[x-\langle x\rangle]<x^{\prime}<\left\langle x^{\prime}\right\rangle+x_{b}^{\prime}+\alpha[x-\langle x\rangle], \\ & \langle x\rangle-x_{b}<x<\langle x\rangle+x_{b}, \\ 0, & \text { otherwise } .\end{cases}
$$

It is clear from Eq. (40) and Fig. 2 that

$$
\begin{aligned}
& \frac{1}{N_{b}} \int d x d x^{\prime} x f_{b}=\langle x\rangle, \\
& \frac{1}{N_{b}} \int d x d x^{\prime} x^{\prime} f_{b}=\left\langle x^{\prime}\right\rangle,
\end{aligned}
$$

as required by the definition of statistical averages in Eq. (8). In the subsequent analysis in Sec. IV, we consider the class of solutions in which the phase space boundaries $x_{b}^{-}(s)$ and $x_{b}^{+}(s)$ in Fig. 2 remain vertical, i.e., there is not an initial perturbation corresponding to a tilt (relative to the vertical) of the boundaries $x_{b}^{-}$and $x_{b}^{+}$in Fig. 2.

\section{A. Evaluation of Macroscopic Quantities and Statistical Averages}

The simple shape of the boundary curves in Fig. 2 and Eq. (40) makes it straightforward to calculate the various macroscopic properties and statistical averages of physical interest. For example, it follows directly from Eq. (40) and Fig. 2 that the density profile $n_{b}(x, s)=$ $\int d x^{\prime} f_{b}\left(x, x^{\prime}, s\right)$ corresponds to the simple step-function profile

$$
n_{b}(x, s)= \begin{cases}0, & -x_{w} \leq x<x_{b}^{-}(s) \\ A \cdot 2 x_{b}^{\prime}(s), & x_{b}^{-}(s)<x<x_{b}^{+}(s) \\ 0, & x_{b}^{+}(s)<x \leq x_{w}\end{cases}
$$

and the area number density $N_{b}=\int d x d x^{\prime} f_{b}\left(x, x^{\prime}, s\right)$ is given by

$$
N_{b}=A \cdot 2 x_{b}^{\prime}(s) \cdot 2 x_{b}(s)=\text { const }
$$

Here, $2 x_{b}(s)$ and $2 x_{b}^{\prime}(s)$ are the thicknesses of the parallelogram in Fig. 2 in the $x$-direction and $x^{\prime}$-direction, respectively. While both $x_{b}(s)$ and $x_{b}^{\prime}(s)$ depend on $s$, it is clear from Eq. (43) that the product $x_{b}(s) x_{b}^{\prime}(s)$ is constant. Furthermore, combining Eqs. (42) and 
(43), the particle number density $n_{b}(s)=2 A x_{b}^{\prime}(s)$ in the interval $-x_{b}<x-\langle x\rangle<x_{b}$ can be expressed as

$$
n_{b}(s)=\frac{N_{b}}{2 x_{b}(s)} .
$$

The average flow velocity $V_{x b}(x, s)=n_{b}^{-1} \int d x^{\prime} x^{\prime} f_{b}\left(x, x^{\prime}, s\right)$ is also straightforward to calculate from Eq. (40) and Fig. 2. Some algebraic manipulation gives

$$
V_{x b}(x, s)=\frac{1}{2}\left[x_{+}^{\prime}(x, s)+x_{-}^{\prime}(x, s)\right]=\left\langle x^{\prime}\right\rangle(s)+\alpha(s)[x-\langle x\rangle(s)],
$$

where $\langle x\rangle(s)$ and $\left\langle x^{\prime}\right\rangle(s)=(d / d s)\langle x\rangle(s)$ are the centroid position and velocity.

To evaluate the statistical averages $\left\langle x^{2}\right\rangle,\left\langle x^{\prime 2}\right\rangle$ and $\left\langle x x^{\prime}\right\rangle$, we make use of the identities (see Sec. II)

$$
\begin{aligned}
\left\langle x^{2}\right\rangle & =\left\langle(x-\langle x\rangle)^{2}\right\rangle+\langle x\rangle^{2}, \\
\left\langle x^{\prime 2}\right\rangle & =\left\langle\left(x^{\prime}-\left\langle x^{\prime}\right\rangle\right)^{2}\right\rangle+\left\langle x^{\prime}\right\rangle^{2}, \\
\left\langle x x^{\prime}\right\rangle & =\left\langle(x-\langle x\rangle)\left(x^{\prime}-\left\langle x^{\prime}\right\rangle\right)\right\rangle+\langle x\rangle\left\langle x^{\prime}\right\rangle=\frac{d}{d s} \frac{1}{2}\left\langle x^{2}\right\rangle,
\end{aligned}
$$

where statistical averages are defined by $\langle\chi\rangle=N_{b}^{-1} \int d x d x^{\prime} \chi f_{b}$. Some straightforward algebraic manipulation that makes use of Eqs. (40) and (43) and Fig. 2 gives the simple expressions

$$
\begin{aligned}
\left\langle(x-\langle x\rangle)^{2}\right\rangle & =\frac{1}{3} x_{b}^{2}(s), \\
\left\langle\left(x^{\prime}-\left\langle x^{\prime}\right\rangle\right)^{2}\right\rangle & =\frac{1}{3}\left[x_{b}^{\prime 2}(s)+\alpha^{2}(s) x_{b}^{2}(s)\right], \\
\left\langle(x-\langle x\rangle)\left(x^{\prime}-\left\langle x^{\prime}\right\rangle\right)\right\rangle & =\frac{1}{3} \alpha(s) x_{b}^{2}(s) .
\end{aligned}
$$

Therefore, from Eq. (47), the mean-square beam thickness $X_{b}^{2}(s)=\left\langle(x-\langle x\rangle)^{2}\right\rangle$ is given by

$$
X_{b}^{2}(s)=\frac{1}{3} x_{b}^{2}(s),
$$

where $x_{b}(s)$ is the half-thickness of the beam. Substituting $\left\langle(x-\langle x\rangle)\left(x^{\prime}-\left\langle x^{\prime}\right\rangle\right)\right\rangle=\alpha(s) X_{b}^{2}(s)$ [Eq. (47)] into Eq. (46) gives

$$
\frac{d}{d s} \frac{1}{2}\left\langle x^{2}\right\rangle=\langle x\rangle\left\langle x^{\prime}\right\rangle+\alpha(s) X_{b}^{2}(s) .
$$

Making use of $\left\langle x^{2}\right\rangle=X_{b}^{2}+\langle x\rangle^{2}$ [Eq. (46)] and $(d / d s)\langle x\rangle=\left\langle x^{\prime}\right\rangle$, Eq. (49) gives directly

$$
\alpha(s)=\frac{1}{X_{b}} \frac{d X_{b}}{d s},
$$


where $X_{b}(s)=(1 / \sqrt{3}) x_{b}(s)$ is the rms beam thickness. Equation (50) determines $\alpha(s)$ in terms of $X_{b}(s)$. Making use of Eqs. (48) and (50), the expressions in Eq. (47) reduce exactly to

$$
\begin{array}{r}
\left\langle(x-\langle x\rangle)^{2}\right\rangle=X_{b}^{2}(s)=\frac{1}{3} x_{b}^{2}(s), \\
\left\langle\left(x^{\prime}-\left\langle x^{\prime}\right\rangle\right)^{2}\right\rangle=\frac{1}{3} x_{b}^{\prime 2}(s)+\left(\frac{d X_{b}}{d s}\right)^{2}, \\
\left\langle(x-\langle x\rangle)\left(x^{\prime}-\left\langle x^{\prime}\right\rangle\right)\right\rangle=X_{b} \frac{d X_{b}}{d s},
\end{array}
$$

for the choice of distribution function in Eq. (40) and Fig. 2.

The unnormalized beam emittance (subtracting out the centroid motion) defined in Eq. (20) is a quantity of considerable physical interest. Making use of Eqs. (20) and (51), we obtain

$$
\begin{aligned}
\frac{1}{4} \epsilon_{x}^{2}(s) & =\left\langle\left(x^{\prime}-\left\langle x^{\prime}\right\rangle\right)^{2}\right\rangle\left\langle(x-\langle x\rangle)^{2}\right\rangle-\left\langle(x-\langle x\rangle)\left(x^{\prime}-\left\langle x^{\prime}\right\rangle\right)\right\rangle^{2} \\
& =\frac{1}{3} x_{b}^{\prime 2} X_{b}^{2}+\left(\frac{d X_{b}}{d s}\right)^{2}-\left(\frac{d X_{b}}{d s}\right)^{2} \\
& =\frac{1}{3} x_{b}^{\prime 2}(s) X_{b}^{2}(s)
\end{aligned}
$$

for the choice of distribution function in Eqs. (40). Substituting $X_{b}^{2}(s)=(1 / 3) x_{b}^{2}(s)$ into Eq. (52), and making use of $2 x_{b}^{\prime}(s) \cdot 2 x_{b}(s)=N_{b} / A=$ const. [Eq. (43)], we obtain

$$
\epsilon_{x}^{2}(s)=\frac{4}{9}\left[x_{b}^{\prime}(s) x_{b}(s)\right]^{2}=\frac{1}{36} \frac{N_{b}^{2}}{A^{2}} \equiv \epsilon_{x 0}^{2}=\text { const. },
$$

where $N_{b}=$ const. is the area number density of beam particles, and $A=$ const. is the phasespace density of beam particles. The fact that $\epsilon_{x}^{2}(s)=\epsilon_{x 0}^{2}=$ const. for the choice of distribution function in Eq. (40) and Fig. 2 leads to enormous simplification in the analysis of the dynamical equation (21) for the evolution of the rms beam thickness $X_{b}(s)=(1 / \sqrt{3}) x_{b}(s)$.

We now turn to a determination of the self-field potential $\psi(x, s)$ required in evaluating the statistical averages $\langle\partial \psi / \partial x\rangle$ and $\langle(x-\langle x\rangle) \partial \psi / \partial x\rangle$ occurring in the rate equations (11) and (21) for the centroid position $\langle x\rangle(s)$ and rms beam thickness $X_{b}(s)$. 


\section{B. Solution for Self-Field Potential $\psi(x, s)$}

We now solve Poisson's equation (37) for the choice of step-function density profile $n_{b}(x, s)$ in Eq. (42), which corresponds to the distribution function $f_{b}\left(x, x^{\prime}, s\right)$ in Eq. (40) and Fig. 2. Making use of Eqs. (42)-(44), Poisson's equation (37) becomes

$$
\frac{\partial^{2} \psi}{\partial x^{2}}= \begin{cases}0, & -x_{w} \leq x<x_{b}^{-}(s) \equiv\langle x\rangle-x_{b}(s) \\ -\frac{2 \pi K_{b}}{N_{b}} n_{b}(s), & x_{b}^{-}(s) \equiv\langle x\rangle-X_{b}(s)<x<x_{b}^{+}(s) \equiv\langle x\rangle+x_{b}(s), \\ 0, & x_{b}^{+}(s) \equiv\langle x\rangle+x_{b}(s)<x \leq x_{w},\end{cases}
$$

where $n_{b}(s)=N_{b} / 2 x_{b}(s)$ is the particle number density. Referring to Fig. 3, Eq. (54) can be solved for $\psi(x, s)$, enforcing the boundary conditions $\psi\left(x= \pm x_{w}, s\right)=0$ at the conducting walls, and continuity of $\psi(x, s)$ and $\partial \psi(x, s) / \partial x$ at the left-most boundary of the density profile, $x_{b}^{-}(s)=\langle x\rangle-x_{b}(s)$, and at the right-most boundary of the density profile, $x_{b}^{+}(s)=\langle x\rangle+x_{b}(s)$, in Fig. 3. For the three regions in Fig. 3, making use of Eq. (54) and $n_{b}(s)=N_{b} / 2 x_{b}(s)$, the solution for $\psi(x, s)$ can be expressed as

$$
\psi(x, s)=\left\{\begin{array}{c}
\psi^{I}(x, s)=\frac{\pi K_{b}}{x_{w}}\left(x+x_{w}\right)\left(x_{w}-\langle x\rangle\right), \\
\text { for }-x_{w} \leq x<x_{b}^{-}(s) \equiv\langle x\rangle-x_{b}(s), \\
\psi^{I I}(x, s)=-\frac{\pi K_{b}}{2 x_{b}}\left[x^{2}+2 \frac{\langle x\rangle}{x_{w}}\left(x_{b}-x_{w}\right) x+\langle x\rangle^{2}+x_{b}^{2}-2 x_{b} x_{w}\right], \\
\text { for } x_{b}^{-}(s) \equiv\langle x\rangle-x_{b}(s)<x<x_{b}^{+}(s) \equiv\langle x\rangle+x_{b}(s), \\
\psi^{I I I}(x, s)=\frac{\pi K_{b}}{x_{w}}\left(x_{w}-x\right)\left(x_{w}+\langle x\rangle\right), \\
\quad \text { for } x_{b}^{+}(s) \equiv\langle x\rangle+x_{b}(s)<x \leq x_{w} .
\end{array}\right.
$$

The solution for $\psi(x, s)$ in Eq. (55) is valid even in the case where the centroid $\langle x\rangle(s)$ undergoes nonlinear motion, provided the beam surfaces do not come in contact with the conducting walls at $x= \pm x_{w}$. Of course Eq. (55) simplifies for the case of a centered beam with $\langle x\rangle=0$.

Of particular interest in the rate equations (11) and (21) for the centroid position $\langle x\rangle(s)$ and rms beam thickness $X_{b}(s)$ are the statistical averages $\langle\partial \psi / \partial x\rangle$ and $\langle(x-\langle x\rangle) \partial \psi / \partial x\rangle$ 


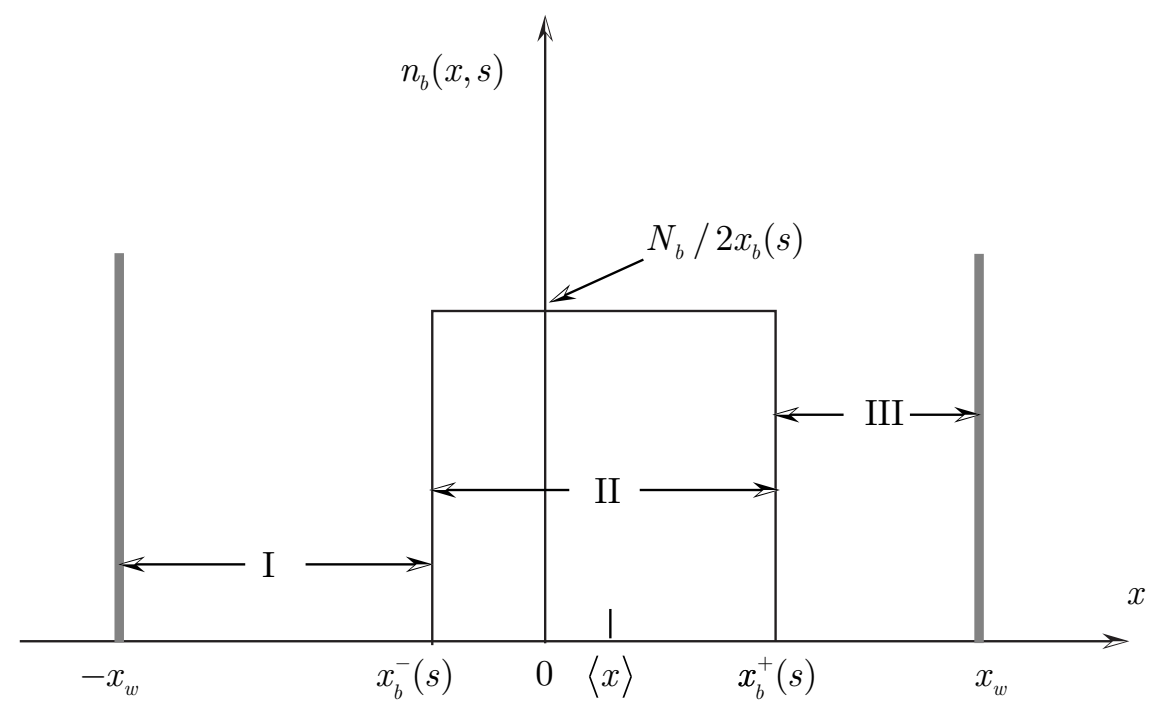

FIG. 3: Plot versus $x$ of the step-function density profile $n_{b}(x, s)$ in Eq. (42) corresponding to the parallelogram distribution in Eq. (40) and Fig. 2. Here, $n_{b}(s)=N_{b} / 2 x_{b}(s)$, and the density profile is centered at $x=\langle x\rangle(s)$.

defined by [see Eq. (8)]

$$
\begin{aligned}
\left\langle\frac{\partial \psi}{\partial x}\right\rangle & =\frac{1}{N_{b}} \int d x d x^{\prime} \frac{\partial \psi}{\partial x} f_{b}=\frac{1}{N_{b}} \int d x \frac{\partial \psi}{\partial x} n_{b} \\
\left\langle(x-\langle x\rangle) \frac{\partial \psi}{\partial x}\right\rangle & =\frac{1}{N_{b}} \int d x d x^{\prime}(x-\langle x\rangle) \frac{\partial \psi}{\partial x} f_{b} \\
& =\frac{1}{N_{b}} \int d x(x-\langle x\rangle) \frac{\partial \psi}{\partial x} n_{b}
\end{aligned}
$$

where $n_{b}(x, s)=\int d x^{\prime} f_{b}\left(x, x^{\prime}, s\right)$ is the number density of beam particles. For the choice of distribution function in Eq. (40) and Fig. 2, the density profile $n_{b}(x, s)$ has the step-function profile in Eq. (42) and Fig. 3, and the expression for $\langle\partial \psi / \partial x\rangle$ in Eq. (56) reduces to

$$
\left\langle\frac{\partial \psi}{\partial x}\right\rangle=\frac{1}{N_{b}} n_{b}(s) \int_{\langle x\rangle-x_{b}(s)}^{\langle x\rangle+x_{b}(s)} d x \frac{\partial}{\partial x} \psi^{I I}(x, s),
$$

where $\psi^{I I}(x, s)$ is defined in Eq. (55), and $n_{b}(s)=N_{b} / 2 x_{b}(s)$ follows from Eq. (44). Substituting Eq. (55) into Eq. (57) readily gives

$$
\left\langle\frac{\partial \psi}{\partial x}\right\rangle=-\frac{\pi K_{b}}{x_{w}}\langle x\rangle
$$

In a similar manner, it can be shown from Eqs. (55) and (56) that

$$
\left\langle(x-\langle x\rangle) \frac{\partial \psi}{\partial x}\right\rangle=-\frac{\pi K_{b}}{3} x_{b}=-\frac{\pi K_{b}}{\sqrt{3}} X_{b},
$$


where use is made of $X_{b}(s)=x_{b}(s) / \sqrt{3}$. The expressions for the statistical averages in Eqs. (58) and (59), valid for the choice of distribution function in Eq. (40) and Fig. 2, can be used directly in the rate equations for $\langle x\rangle(s)$ and $X_{b}(s)$ in Eqs. (11) and (21).

\section{Rate Equations for $\langle x\rangle(s)$ and $X_{b}(s)$}

We substitute Eqs. (58) and (59) into the rate equations (11) and (21) for the motion of the beam centroid $\langle x\rangle(s)$ and rms beam thickness $X_{b}(s)=\left\langle(x-\langle x\rangle)^{2}\right\rangle^{1 / 2}$. This gives

$$
\frac{d^{2}}{d s^{2}}\langle x\rangle+\left[\kappa_{x}(s)-\frac{\pi K_{b}}{x_{w}}\right]\langle x\rangle=0,
$$

and

$$
\frac{d^{2}}{d s^{2}} X_{b}+\left[\kappa_{x}(s)-\frac{\pi K_{b}}{\sqrt{3} X_{b}}\right] X_{b}=\frac{\epsilon_{x 0}^{2}}{4 X_{b}^{3}},
$$

where $\epsilon_{x 0}$ is the constant emittance defined in Eq. (53). Note from Eqs. (58) and (60) that the self-field force on the beam centroid, $-\langle\partial \psi / \partial x\rangle=\left(\pi K_{b} / x_{w}\right)\langle x\rangle$, is always defocusing and is proportional to the displacement $\langle x\rangle$ from the center position $(x=0)$, and the constant factor $\pi K_{b} / x_{w}$. Furthermore, the self-field force in Eq. (60) is associated with image charges in the conducting wall. In particular, at fixed beam intensity $\left(K_{b}\right)$, the self-field force in Eq. (60) becomes negligibly small as $x_{w} \rightarrow \infty$. On the other hand, from Eqs. (59) and (61), the self-field force term is $-X_{b}^{-1}\langle(x-\langle x\rangle) \partial \psi / \partial x\rangle=\pi K_{b} / \sqrt{3}=$ const., which is also defocusing, but is independent of $X_{b}$ and $\langle x\rangle$.

Equations (60) and (61) constitute closed dynamical equations for the motion of the beam centroid $\langle x\rangle(s)$ and rms beam thickness $X_{b}(s)$. Moreover, Eqs. (60) and (61) can be integrated numerically for a wide range of choices of lattice function $\kappa_{x}(s)$, beam emittance $\epsilon_{x 0}$, beam intensity $K_{b}$, and conducting wall location $x_{w}$. Note from Eq. (60) that if the beam is initially centered with $\langle x\rangle(s=0)=0=[d\langle x\rangle / d s]_{s=0}$, then $\langle x\rangle=0=(d / d s)\langle x\rangle$ at all subsequent $s$. Most importantly, solving Eqs. (60) and (61) for $\langle x\rangle(s)$ and $X_{b}(s)$ is fully equivalent to solving the nonlinear Vlasov-Maxwell equations (1) and (2) for the choice of parallelogram distribution in Eq. (40) and Fig. 2 with constant phase-space density $f_{b}\left(x, x^{\prime}, s\right)=A=$ const. Making the identifications $x_{b}(s)=\sqrt{3} X_{b}(s)$ [Eq. (51)], $\alpha(s)=$ $X_{b}^{-1} d X_{b} / d s$ [Eq. (50)], $x_{b}^{\prime}(s)=(\sqrt{3} / 2) \epsilon_{x 0} / X_{b}(s)$ [Eq. (53)], and $\left\langle x^{\prime}\right\rangle(s)=(d / d s)\langle x\rangle(s)$ [Eq. (9)], it follows directly from Eq. (39) that the phase-space boundaries in Eqs. (39), (40) 


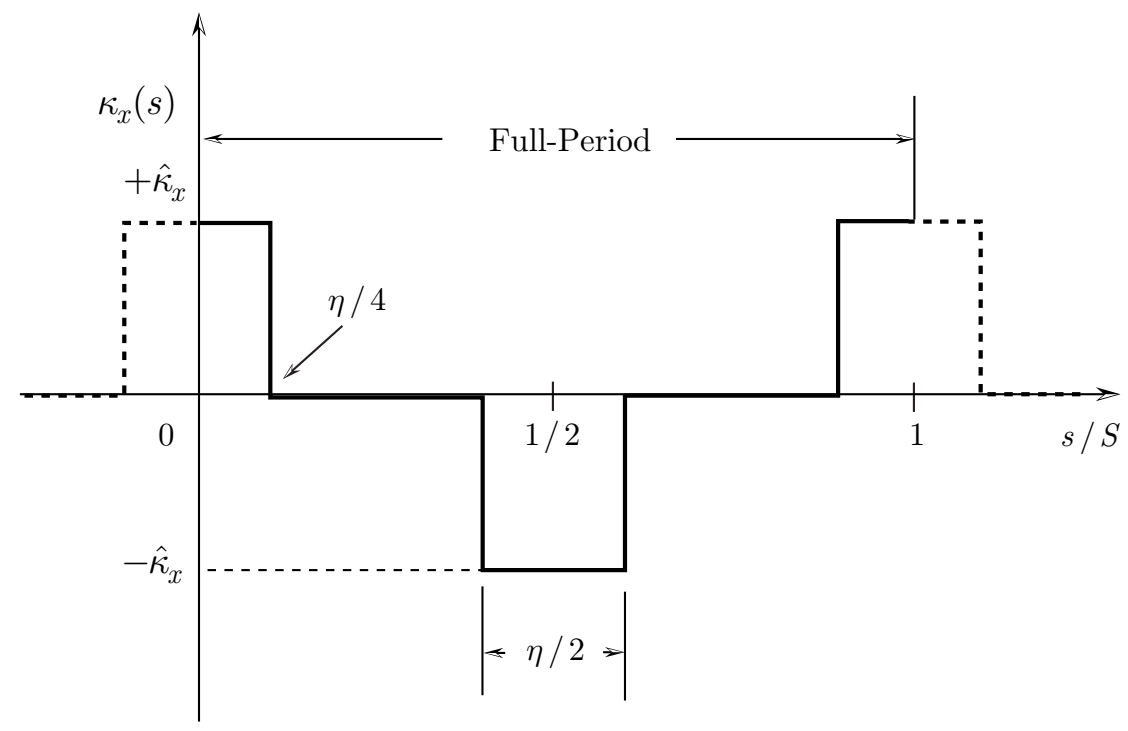

FIG. 4: Plot of lattice function $\kappa_{x}(s)$ versus $s / S$ for a periodic step-function lattice with amplitude $\hat{\kappa}_{x}=$ const. and filling factor $\eta$.

and Fig. 2 are given by

$$
\begin{aligned}
x_{ \pm}^{\prime}(x, s) & =\frac{d}{d s}\langle x\rangle(s) \pm \frac{\sqrt{3}}{2} \frac{\epsilon_{x 0}}{X_{b}(s)}+\frac{1}{X_{b}} \frac{d X_{b}}{d s}(x-\langle x\rangle(s)), \\
x_{b}^{ \pm}(s) & =\langle x\rangle(s) \pm \sqrt{3} X_{b}(s) .
\end{aligned}
$$

Therefore, a determination of $\langle x\rangle(s)$ and $X_{b}(s)$ from Eqs. (60) and (61) fully specifies the distribution function in Eq. (40) and Fig. 2. For matched-beam solutions $X_{b}(s+S)=X_{b}(s)$ to the nonlinear rms envelope equation (61), the shape of the parallelogram in Fig. 2 pulsates with period $S$ in a frame of reference centered at $\left(\langle x\rangle,\left\langle x^{\prime}\right\rangle\right)$. Depending on the initial conditions for $\langle x\rangle(s=0)$ and $[(d / d s)\langle x\rangle]_{s=0}$, however, the motion of the centroid $\left(\langle x\rangle,\left\langle x^{\prime}\right\rangle\right)$ in Fig. 2 can be more irregular, with both fast-oscillatory and slow-oscillatory components.

For present purposes, we examine Eqs. (60) and (61) for the choice of a periodic stepfunction lattice $\kappa_{x}(s+S)=\kappa_{x}(s)$ illustrated in Fig. 4. Here, $\hat{\kappa}_{x}=$ const. is the lattice amplitude, and $\eta$ is the filling factor. For simplicity we consider here a centered beam with $[\langle x\rangle]_{s=0}=[d\langle x\rangle / d s]_{s=0}=0$, so that $\langle x\rangle=d\langle x\rangle / d s=0$ at all subsequent $s$. Typical numerical results obtained from Eq. (61) for the rms beam thickness $X_{b}(s)$ are shown in Fig. 5, where $X_{b}(s) / \sqrt{\epsilon_{x 0} S}$ is plotted versus $s / S$ for a matched beam with moderate spacecharge intensity propagating through the periodic step-function lattice in Fig. 4. Here, the dimensionless system parameters correspond to $\widehat{\kappa}_{x} S^{2}=14.92$, filling factor $\eta=0.3$, and 


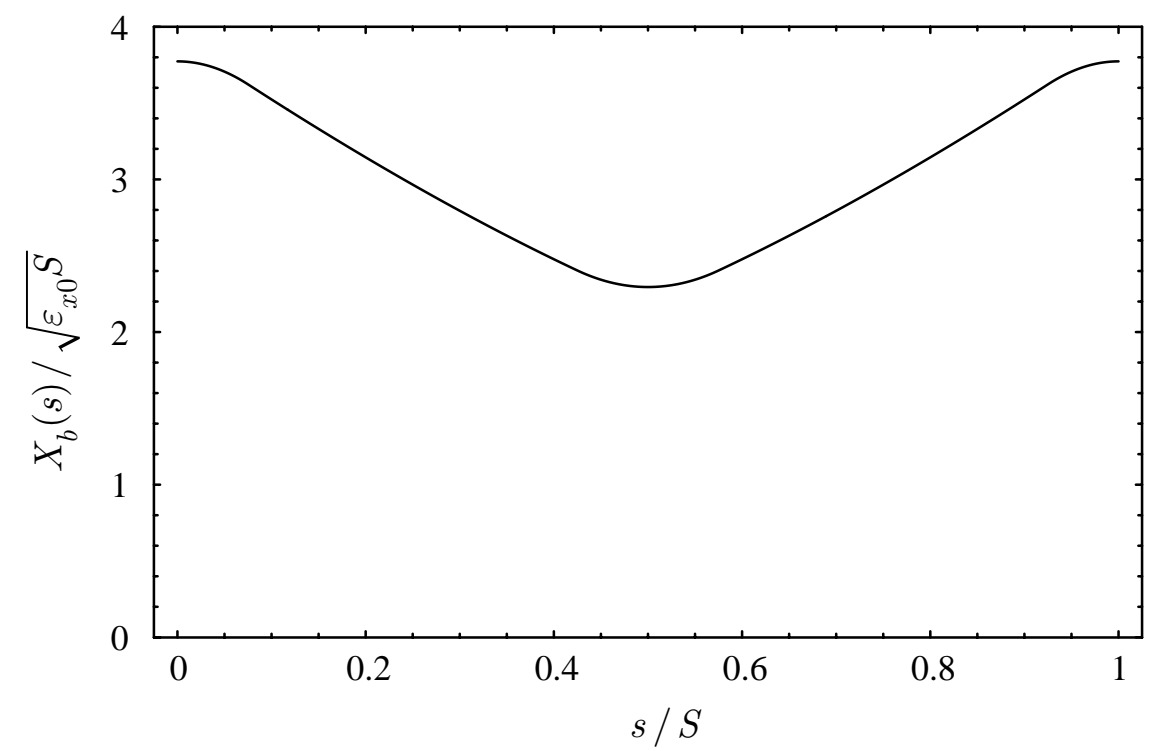

FIG. 5: Illustrative plot of $X_{b}(s) / \sqrt{\epsilon_{x 0} S}$ versus $s / S$ obtained numerically from Eq. (61) for a matched beam with moderate space-charge intensity propagating through the periodic stepfunction lattice in Fig. 4. Here, the dimensionless system parameters correspond to $\hat{\kappa}_{x} S^{2}=14.92$, $\eta=0.3$, and $K_{b} S / \sqrt{\epsilon_{x 0} S}=5$, with vacuum phase advance $\sigma_{v a c}=60^{\circ}$ and depressed phase advance $\sigma=3.4^{\circ}$.

normalized beam intensity $K_{b} S / \sqrt{\epsilon_{x 0} S}=5.0$. Moreover, the vacuum phase advance per lattice period corresponds to $\sigma_{v a c}=\lim _{K_{b} \rightarrow 0}\left(\epsilon_{x 0} / 2\right) \int_{s_{0}}^{s_{0}+S} d s / X_{b}^{2}(s)=60^{\circ}$, and the depressed phase advance $\left(K_{b} \neq 0\right)$ corresponds to $\sigma=3.4^{\circ}$. Note from Fig. 5 that the solution for $X_{b}(s)$ corresponds to a matched beam with $X_{b}(s+S)=X_{b}(s)$, and the corresponding phasespace boundaries in Eq. (62) correspond to a periodically-focused beam equilibrium with $x_{ \pm}^{\prime}(x, s+S)=x_{ \pm}^{\prime}(x, s)$ and $x_{b}^{ \pm}(s+S)=x_{b}^{ \pm}(s)$.

\section{LAGRANGIAN DESCRIPTION OF NONLINEAR BEAM DYNAMICS}

For the case of uniform phase space density, it was shown in Sec. III.B that the basic dynamical equations for a planar sheet beam propagating through a periodic focusing field could be cast into the form of the macroscopic warm-fluid equations (35)-(37) with tripleadiabatic pressure relation $P_{b}(x, s)=\left(\hat{P}_{b} / \hat{n}_{b 0}^{3}\right) n_{b}^{3}(x, s)$ and zero heat flow $Q_{b}(x, s)=0$. Introducing the (normalized) electric field $E_{x}(x, s)=-\partial \psi(x, s) / \partial x$ and making use of 
Eqs. (35)-(37), it follows that the number density $n_{b}(x, s)=\int d x^{\prime} f_{b}$ and (normalized) average velocity $V_{x b}(x, s)=\left(\int d x^{\prime} x^{\prime} f_{b}\right) /\left(\int d x^{\prime} f_{b}\right)$ evolve exactly according to

$$
\begin{gathered}
\left(\frac{\partial}{\partial s}+V_{x b} \frac{\partial}{\partial x}\right) n_{b}+n_{b} \frac{\partial V_{x b}}{\partial x}=0 \\
\left(\frac{\partial}{\partial s}+V_{x b} \frac{\partial}{\partial x}\right) V_{x b}+\frac{3}{2} v_{T b}^{2} \frac{\partial}{\partial x} n_{b}^{2}=-\kappa_{x}(s) x+E_{x}
\end{gathered}
$$

where $E_{x}(x, s)$ solves

$$
\frac{\partial E_{x}}{\partial x}=\frac{2 \pi K_{b}}{N_{b}} n_{b}
$$

Here, $v_{T b}^{2} \equiv \hat{P}_{b} / \hat{n}_{b 0}^{3}=$ const. is the normalized thermal speed. As noted in Sec. III, for uniform phase-space density, Eqs. (63)-(65) are exactly equivalent to the Vlasov-Maxwell equations (1) and (2), and Eqs. (27)-(29) for $\psi(x, s)$ and the phase-space boundary curves, $x_{+}^{\prime}(x, s)$ and $x_{-}^{\prime}(x, s)$.

\section{A. Dynamical Equations in Lagrangian Variables}

The fluid-Maxwell equations (63)-(65) are particularly amenable to analysis in Lagrangian variables following the motion of a fluid element $[35,36]$. We introduce the Lagrangian variables $\left(x_{0}, \tau\right)$ following a fluid element defined by

$$
\begin{aligned}
\tau & =s, \\
x_{0} & =x-\int_{0}^{\tau} d \tau^{\prime} V_{x b}\left(x_{0}, \tau^{\prime}\right) .
\end{aligned}
$$

Here, $\tau$ is a (normalized) time variable, and it follows exactly from Eq. (66) that derivatives transform according to

$$
\begin{array}{r}
\frac{\partial}{\partial x}=\left[1+\int_{0}^{\tau} d \tau^{\prime} \frac{\partial}{\partial x_{0}} V_{x b}\left(x_{0}, \tau^{\prime}\right)\right]^{-1} \frac{\partial}{\partial x_{0}} \\
\frac{\partial}{\partial s}=\frac{\partial}{\partial \tau}-V_{x b}\left(x_{0}, \tau\right)\left[1+\int_{0}^{\tau} d \tau^{\prime} \frac{\partial}{\partial x_{0}} V_{x b}\left(x_{0}, \tau^{\prime}\right)\right]^{-1} \frac{\partial}{\partial x_{0}} .
\end{array}
$$

From Eq. (67) we obtain

$$
\frac{\partial}{\partial s}+V_{x b} \frac{\partial}{\partial x}=\frac{\partial}{\partial \tau}
$$

and the continuity equation (63) in Lagrangian variables becomes

$$
\frac{\partial}{\partial \tau} n_{b}\left(x_{0}, \tau\right)+\frac{n_{b}\left(x_{0}, \tau\right)}{\left[1+\int_{0}^{\tau} d \tau^{\prime} \partial V_{x b}\left(x_{0}, \tau^{\prime}\right) / \partial x_{0}\right]} \frac{\partial}{\partial x_{0}} V_{x b}\left(x_{0}, \tau\right)=0 .
$$


Equation (69) can be integrated exactly with respect to $\tau$ to give

$$
n_{b}\left(x_{0}, \tau\right)=\frac{n_{b}\left(x_{0}, 0\right)}{\left[1+\int_{0}^{\tau} d \tau^{\prime} \partial V_{x b}\left(x_{0}, \tau^{\prime}\right) / \partial x_{0}\right]} .
$$

Note that Eq. (70) gives a closed expression for $n_{b}\left(x_{0}, \tau\right)$ in Lagrangian variables in terms of $n_{b}\left(x_{0}, 0\right)$ and $V_{x b}\left(x_{0}, \tau\right)$.

Poisson's equation (65) also simplifies in Lagrangian variables. Making use of Eq. (67) we obtain

$$
\frac{1}{\left[1+\int_{0}^{\tau} d \tau^{\prime} \partial V_{x b}\left(x_{0}, \tau^{\prime}\right) / \partial x_{0}\right]} \frac{\partial}{\partial x_{0}} E_{x}\left(x_{0}, \tau\right)=\frac{2 \pi K_{b}}{N_{b}} n_{b}\left(x_{0}, \tau\right) .
$$

where $n_{b}\left(x_{0}, \tau\right)$ is given by Eq. (70). Equations (70) and (71) readily give

$$
\frac{\partial}{\partial x_{0}} E_{x}\left(x_{0}, \tau\right)=\frac{2 \pi K_{b}}{N_{b}} n_{b}\left(x_{0}, 0\right)
$$

A very important consequence of transforming to Lagrangian variables is evident from Eq. (72). In particular, $E_{x}\left(x_{0}, \tau\right)=E_{x}\left(x_{0}, 0\right)$ is independent of $\tau$, and depends only on the initial density profile $n_{b}\left(x_{0}, 0\right)$.

We now return to the force balance equation (64), transforming to Lagrangian variables according to (67), (68) and (70). This gives (exactly)

$$
\begin{aligned}
\frac{\partial}{\partial \tau} V_{x b}\left(x_{0}, \tau\right) & +\frac{3}{2} \frac{v_{T b}^{2}}{\left[1+\int_{0}^{\tau} d \tau^{\prime} \partial V_{x b}\left(x_{0}, \tau^{\prime}\right) / \partial x_{0}\right]} \\
& \times \frac{\partial}{\partial x_{0}}\left\{\frac{n_{b}\left(x_{0}, 0\right)}{\left[1+\int_{0}^{\tau} d \tau^{\prime} \partial V_{x b}\left(x_{0}, \tau^{\prime}\right) / \partial x_{0}\right]}\right\}^{2}=E_{x}\left(x_{0}, \tau\right) \\
& -\kappa_{x}(\tau)\left[x_{0}+\int_{0}^{\tau} d \tau^{\prime} V_{x b}\left(x_{0}, \tau^{\prime}\right)\right]
\end{aligned}
$$

where $E_{x}\left(x_{0}, \tau\right)$ is determined self-consistently in terms of $n_{b}\left(x_{0}, 0\right)$ from Eq. (72). Operating on Eq. (73) with $\partial / \partial x_{0}$ and rearranging terms, we readily obtain

$$
\begin{array}{r}
\frac{\partial^{2}}{\partial \tau^{2}}\left[1+\int_{0}^{\tau} d \tau^{\prime} \frac{\partial}{\partial x_{0}} V_{x b}\left(x_{0}, \tau^{\prime}\right)\right]+\kappa_{x}(\tau)\left[1+\int_{0}^{\tau} d \tau^{\prime} \frac{\partial}{\partial x_{0}} V_{x b}\left(x_{0}, \tau^{\prime}\right)\right] \\
+v_{T b}^{2} \frac{\partial}{\partial x_{0}}\left[\frac{1}{n_{b}\left(x_{0}, 0\right)} \frac{\partial}{\partial x_{0}}\left\{\frac{n_{b}\left(x_{0}, 0\right)}{\left[1+\int_{0}^{\tau} d \tau^{\prime} \partial V_{x b}\left(x_{0}, \tau^{\prime}\right) / \partial x_{0}\right]}\right\}^{3}\right]=\frac{2 \pi K_{b}}{N_{b}} n_{b}\left(x_{0}, 0\right) .
\end{array}
$$

Equation (74) is a closed, partial differential equation for the density compression factor

$$
\frac{n_{b}\left(x_{0}, 0\right)}{n_{b}\left(x_{0}, \tau\right)}=1+\int_{0}^{\tau} d \tau^{\prime} \frac{\partial}{\partial x_{0}} V_{x b}\left(x_{0}, \tau^{\prime}\right)
$$


in Lagrangian variables, which is fully equivalent to the original dynamical equations (63)(65) in laboratory-frame variables. Substituting Eq. (75) into Eq. (74) gives directly

$$
\begin{gathered}
\frac{\partial^{2}}{\partial \tau^{2}}\left[\frac{1}{n_{b}\left(x_{0}, \tau\right)}\right]+\kappa_{x}(\tau)\left[\frac{1}{n_{b}\left(x_{0}, \tau\right)}\right] \\
+\frac{v_{T b}^{2}}{n_{b}\left(x_{0}, 0\right)} \frac{\partial}{\partial x_{0}}\left[\frac{1}{n_{b}\left(x_{0}, 0\right)} \frac{\partial}{\left.\partial x_{0}\right)}\left\{n_{b}\left(x_{0}, \tau\right)\right\}^{3}\right]=\frac{2 \pi K_{b}}{N_{b}}
\end{gathered}
$$

in the region where $n_{b}\left(x_{0}, 0\right)$ is non-zero.

Equation (76) [or equivalently, Eq. (74)] constitutes the final dynamical equation in Lagrangian variables, and can be used to investigate the detailed nonlinear dynamics of intense beam propagation for a wide variety of input density profiles $n_{b}\left(x_{0}, 0\right)$, lattice functions $\kappa_{x}(\tau)$, normalized beam intensity $\left(K_{b}\right)$, and beam emittance (proportional to $v_{T b}^{2}$ ). Furthermore, Eq. (76) is well-posed as an initial-value problem. For specified $n_{b}\left(x_{0}, 0\right)$ and $V_{x b}\left(x_{0}, 0\right)$, it follows from Eq. (75) that

$$
\left.\frac{\partial}{\partial \tau} n_{b}\left(x_{0}, \tau\right)\right|_{\tau=0}=-n_{b}\left(x_{0}, 0\right) \frac{\partial}{\partial x_{0}} V_{x b}\left(x_{0}, 0\right),
$$

and Eq. (76) can generally be integrated numerically to determine $n_{b}\left(x_{0}, \tau\right)$ once the initial profiles for $n_{b}\left(x_{0}, 0\right)$ and $V_{x b}\left(x_{0}, 0\right)$ and beam parameters are specified.

Once $n_{b}\left(x_{0}, \tau\right)$ is determined from Eq. (76), the expression for $n_{b}\left(x_{0}, 0\right) / n_{0}\left(x_{0}, \tau\right)$ can be used to formally determine the inverse transformation to laboratory-frame variables $(x, s)$ defined in Eq. (66). For example, consider the case where the initial density profile is an even function of $x_{0}$ with $n_{b}\left(-x_{0}, 0\right)=n_{b}\left(x_{0}, 0\right)$, and the initial flow velocity profile is an odd function of $x_{0}$ with $V_{x b}\left(-x_{0}, 0\right)=-V_{x b}\left(x_{0}, 0\right)$ and $V_{x b}\left(x_{0}=0,0\right)=0$. It readily follows from Eqs. (74)-(76) that

$$
\begin{gathered}
n_{b}\left(-x_{0}, \tau\right)=n_{b}\left(x_{0}, \tau\right), \\
V_{x b}\left(-x_{0}, \tau\right)=-V_{x b}\left(x_{0}, \tau\right),
\end{gathered}
$$

where $V_{x b}\left(x_{0}=0, \tau\right)=0$ for all values of $\tau$. Integrating Eq. (75) with respect to $x_{0}$ then gives

$$
x_{0}+\int_{0}^{\tau} d \tau^{\prime} V_{x b}\left(x_{0}, \tau^{\prime}\right)=\int_{0}^{x_{0}} d x_{0} \frac{n_{b}\left(x_{0}, 0\right)}{n_{b}\left(x_{0}, \tau\right)},
$$

which is required to determine the inverse transformation from Lagrangian variables $\left(x_{0}, \tau\right)$ to laboratory-frame variables $(x, s)$ in Eq. (66). 


\section{B. Cold-Beam Limit}

As noted earlier, Eq. (76) [or equivalently, Eq. (74)] can be used to describe in Lagrangian variables the beam dynamics for a wide variety of initial profiles and system parameters. For present purposes, we consider the special case of a cold beam with negligible transverse emittance, i.e.,

$$
v_{T b}^{2} \rightarrow 0
$$

In this case, Eq. (76) simplifies to become

$$
\frac{\partial^{2}}{\partial \tau^{2}}\left[\frac{1}{n_{b}\left(x_{0}, \tau\right)}\right]+\kappa_{x}(\tau)\left[\frac{1}{n_{b}\left(x_{0}, \tau\right)}\right]=\frac{2 \pi K_{b}}{N_{b}} .
$$

Note that Eq. (81) is an inhomogeneous, linear, ordinary differential equation for $1 / n_{b}\left(x_{0}, \tau\right)$, with $x_{0}$ occurring as a continuous parameter. Indeed, Eq. (81) can be integrated numerically with respect to $\tau$ for a wide variety of periodic lattice functions $\kappa_{x}(\tau+S)=\kappa_{x}(\tau)$, and initial density profiles $n_{b}\left(x_{0}, 0\right)$ consistent with Eq. (77).

A useful representation of the general solution to Eq. (81) is

$$
\frac{1}{n_{b}\left(x_{0}, \tau\right)}=A\left(x_{0}, \tau\right) w\left(x_{0}, \tau\right) \cos \psi\left(x_{0}, \tau\right)+B\left(x_{0}, \tau\right) w\left(x_{0}, \tau\right) \sin \psi\left(x_{0}, \tau\right) .
$$

In Eq. (82), the envelope function $w\left(x_{0}, \tau\right)$ is taken to solve

$$
\frac{\partial^{2}}{\partial \tau^{2}} w\left(x_{0}, \tau\right)+\kappa_{x}(\tau) w\left(x_{0}, \tau\right)=\frac{1}{w^{3}\left(x_{0}, \tau\right)}
$$

and the phase function $\psi\left(x_{0}, \tau\right)$ is defined by

$$
\psi\left(x_{0}, \tau\right)=\int_{0}^{\tau} \frac{d \tau^{\prime}}{w^{2}\left(x_{0}, \tau^{\prime}\right)} .
$$

Substituting Eqs. (82)-(84) into Eq. (81), we obtain

$$
\frac{1}{w}\left[2 \frac{\partial B}{\partial \tau}+\frac{\partial}{\partial \tau}\left(\frac{\partial A}{\partial \tau} w^{2}\right)\right] \cos \psi+\frac{1}{w}\left[-2 \frac{\partial A}{\partial \tau}+\frac{\partial}{\partial \tau}\left(\frac{\partial B}{\partial \tau} w^{2}\right)\right] \sin \psi=\frac{2 \pi K_{b}}{N_{b}}
$$

where we have suppressed the $\left(x_{0}, \tau\right)$ arguments in Eq. (85). It is readily shown that Eq. (85) is satisfied exactly provided the amplitudes $A\left(x_{0}, \tau\right)$ and $B\left(x_{0}, \tau\right)$ solve

$$
\begin{aligned}
& \frac{\partial A}{\partial \tau}=-\frac{2 \pi K_{b}}{N_{b}} w \sin \psi, \\
& \frac{\partial B}{\partial \tau}=\frac{2 \pi K_{b}}{N_{b}} w \cos \psi .
\end{aligned}
$$


Equations (83), (84) and (86) can be used to determine $w\left(x_{0}, \tau\right), A\left(x_{0}, \tau\right)$ and $B\left(x_{0}, \tau\right)$, and therefore the solution for $1 / n_{b}\left(x_{0}, \tau\right)$ in Eq. (82). Some straightforward algebra that makes use of Eqs. (77), (82), (84) and (86) shows that the appropriate initial conditions at $\tau=0$ are given by

$$
\begin{aligned}
\frac{1}{n_{b}\left(x_{0}, 0\right)} & =[A w]_{\tau=0} \\
\frac{\partial}{\partial x_{0}} V_{x b}\left(x_{0}, 0\right) & =-\left[\frac{1}{w} \frac{\partial w}{\partial \tau}+\frac{B}{A w^{2}}\right]_{\tau=0} .
\end{aligned}
$$

For specified initial conditions, once the solutions for $w\left(x_{0}, \tau\right), A\left(x_{0}, \tau\right)$ and $B\left(x_{0}, \tau\right)$ are obtained from Eqs. (83), (84) and (86), the solution for $n\left(x_{0}, \tau\right)$ can be determined from Eq. (82), and the inverse transformation to laboratory-frame variables $(x, s)$ obtained from Eqs. (66) and (79).

\section{Examples in Cold-Beam Limit}

The Lagrangian formulation developed in Secs. V.A and V.B can be applied to a wide variety of intense beam profiles $n_{b}\left(x_{0}, 0\right)$ and $V_{x b}\left(x_{0}, 0\right)$. To illustrate the power of the Lagrangian formalism in analyzing the beam dynamics, we consider here two simple examples in the cold-beam limit.

The first example corresponds to a periodic step-function lattice in which $\kappa_{x}(\tau+s)=$ $\kappa_{x}(\tau)$ has the waveform illustrated in Fig. 4 with filling factor $\eta$ and constant amplitude $\widehat{\kappa}_{x}$. In this case, the periodic solutions $w_{x}(\tau+s)=w_{x}(\tau)$ obtained (numerically) from Eq. (83) have vacuum phase advance $\sigma_{v a c}$ determined from

$$
\sigma_{v a c}=\int_{s_{0}}^{s_{0}+S} \frac{d \tau}{w^{2}}
$$

Moreover, the corresponding value of the (approximate) smooth-focusing coefficient $\kappa_{s f}$ is given by [1]

$$
\kappa_{s f} S^{2}=\frac{1}{16} \eta^{2}\left(1-\frac{2}{3} \eta\right) \widehat{\kappa}_{x}^{2} S^{4}
$$

In the first example, we consider the choice of initial density profile at $\tau=0$ corresponding 
to

$$
n_{b}\left(x_{0}, 0\right)= \begin{cases}\widehat{n}_{b}\left[1+\Delta\left(1-x_{0}^{2} / x_{b 0}^{2}\right)\right], & 0 \leq\left|x_{0}\right|<x_{b 0} \\ 0, & \left|x_{0}\right|>x_{b 0} .\end{cases}
$$

Here, $\widehat{n}_{b}=n_{b}\left(x_{0}= \pm x_{b 0}, 0\right)$ is the initial edge density of the sheet beam, and $\Delta$ is a (dimensionless) measure of the amplitude of the initial density perturbation at $x_{0}=0$, with $n_{b}\left(x_{0}=0,0\right)=\widehat{n}_{b}(1+\Delta)$. It is further assumed that $V_{x b}\left(x_{0}, 0\right)=0$, and therefore $n_{b}\left(x_{0}, \tau\right)$ and $V_{x b}\left(x_{0}, \tau\right)$ evolve according to the symmetries in Eq. (78). Finally, we introduce the (dimensionless) measure of normalized beam intensity $s_{b}$ defined by

$$
s_{b}=\frac{\widehat{\omega}_{p b}^{2}}{\gamma_{b}^{2} \omega_{\beta \perp}^{2}}
$$

where $\widehat{\omega}_{p b}^{2}=4 \pi \widehat{n}_{b} e_{b}^{2} / \gamma_{b} m_{b}$ is the relativistic plasma frequency-squared, and $\omega_{\beta \perp}=\sqrt{\kappa_{s f}} \beta_{b} c$ is the (smooth-focusing) betatron frequency. Then, making use of the definition of $K_{b}$ in Eq. (5), the dynamical equation $(81)$ for $n_{b}\left(x_{0}, \tau\right)$ in Lagrangian variables can be expressed (exactly) in the equivalent form

$$
S^{2} \frac{\partial^{2}}{\partial \tau^{2}}\left[\frac{\widehat{n}_{b}}{n_{b}\left(x_{0}, \tau\right)}\right]+\kappa_{x}(\tau) S^{2}\left[\frac{\widehat{n}_{b}}{n_{b}\left(x_{0}, \tau\right)}\right]=s_{b} \kappa_{s f} S^{2}
$$

As a numerical example corresponding to the step-function lattice in Fig. 4 and the initial density profile in Eq. (90), we consider the choice of system parameters

$$
\begin{array}{r}
\widehat{\kappa}_{q} S^{2}=14.92, \quad \eta=0.3, \quad \sigma_{v a c}=60^{\circ}, \\
\kappa_{s f} S^{2}=1.0, \quad s_{b}=1, \quad \Delta=-0.2 .
\end{array}
$$

Note from Eq. (93) that the beam has large intensity close to the space-charge limit $\left(s_{b}=1\right)$, and that the density perturbation (relative to the uniform beam density) has moderately large amplitude $(\Delta=-0.2)$. The numerical results corresponding to Eq. (93) are illustrated in Figs. $6-8$. In Fig. 6, the profile for $n_{b}\left(x_{0}, \tau\right)$ obtained numerically from Eqs. (82) (86) is plotted versus $x_{0}$ and $\tau$. Note that $n_{b}\left(x_{0}, \tau\right)=n_{b}\left(-x_{0}, \tau\right)$ evolves symmetrically, as expected from Eqs. (78) and (90). In Lagrangian variables, it is evident from Fig. 6 that the layer maintains constant thickness $2 x_{b 0}$, but undergoes strong oscillatory modulation as a function of $\tau / S$, and over the layer cross-section as a function of $x_{0} / x_{b 0}$. From Eqs. (66) and 


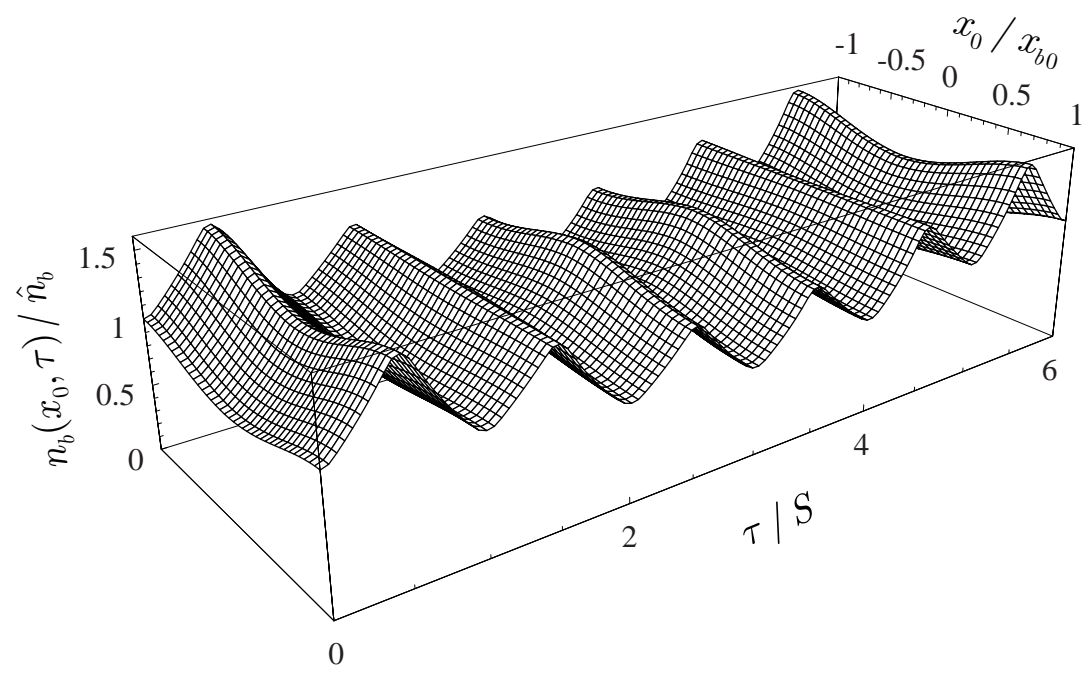

FIG. 6: Plot of density profile $n_{b}\left(x_{0}, \tau\right)$ in Lagrangian variables obtained numerically from Eqs. (82) - (86) for the initial density profile in Eq. (90) and choice of system parameters in Eq. (93).

(79), the corresponding back-transformation to laboratory-frame variables $(x, s)$ consistent with Eq. (92) and Fig. 6 is determined from

$$
x\left(x_{0}, s\right)=\int_{0}^{x_{0}} d x_{0}^{\prime} \frac{n_{b}\left(x_{0}^{\prime}, 0\right)}{n_{b}\left(x_{0}^{\prime}, s\right)} .
$$

For example, at the layer edge $\pm x_{b}(s)$, Fig. 7 shows a plot of $x_{b}(s) \equiv x\left(x_{b 0}, s\right)$ obtained numerically from Eqs. (82) - (86) and Eq. (93). Note from Fig. 7 that the layer edge in the laboratory frame, $\pm x_{b}(s)$, has a fast oscillatory modulation with period equal to the lattice period $S$, plus a slow oscillatory modulation with period approximately equal to $2 \pi / \sqrt{\kappa_{s f}}=2 \pi S$. Finally, making use of Eq. (70), or equivalently, the numerical solution for $n_{b}\left(x_{0}, \tau\right)$ obtained from Eqs. $(82)-(86)$, with $\tau=s$ and $x_{0}=x_{0}(x, s)$, the density profile $n_{b}(x, \tau)$ in laboratory-frame variables $(x, s)$ is illustrated in Fig. 8, which clearly shows the large-amplitude modulation of the density profile in the laboratory frame.

As a second example, we adopt a smooth-focusing model in which the lattice function $\kappa_{x}(\tau)$ is replaced by the constant value $\kappa_{s f}=$ const in Eq. (81), or equivalently, in (92). In this case, Eq. (92) is exactly integrable for general initial density profile $n_{b}\left(x_{0}, 0\right)$. Assuming initial conditions with $n_{b}\left(-x_{0}, 0\right)=n_{b}\left(x_{0}, 0\right)$ and $V_{x b}\left(-x_{0}, 0\right)=-V_{x b}\left(x_{0}, 0\right)$, it follows exactly from Eqs. (75) and (92) that the solutions for $n_{b}\left(x_{0}, \tau\right)$ and $V_{x b}\left(x_{0}, \tau\right)$ can be expressed as

$$
n_{b}\left(x_{0}, \tau\right)=\frac{n_{b}\left(x_{0}, 0\right)}{s_{b} n_{b}\left(x_{0}, 0\right) / \widehat{n}_{b}+\left[1-s_{b} n_{b}\left(x_{0}, 0\right) / \widehat{n}_{b}\right] \cos \left(k_{\beta} \tau\right)},
$$




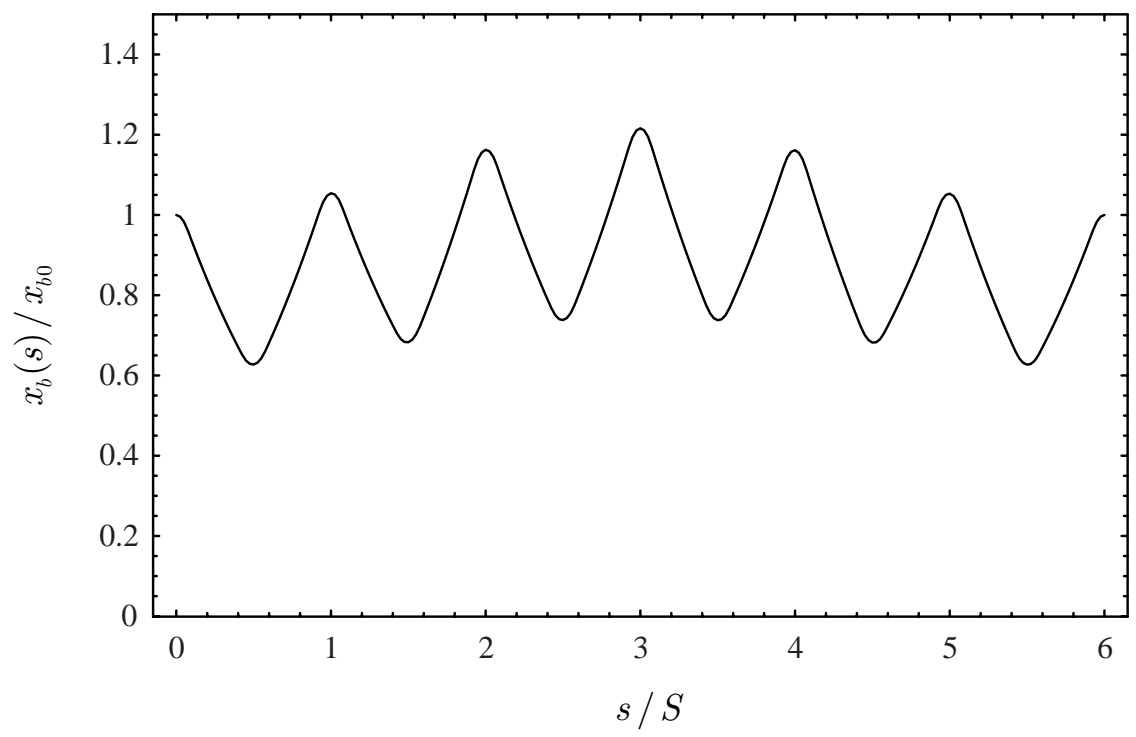

FIG. 7: Plot of layer edge location $x_{b}(s)=x\left(x_{b 0}, s\right)$ obtained numerically from Eqs. (82) - (86) and Eq. (94) for the initial density profile in Eq. (90) and choice of system parameters in Eq. (93).

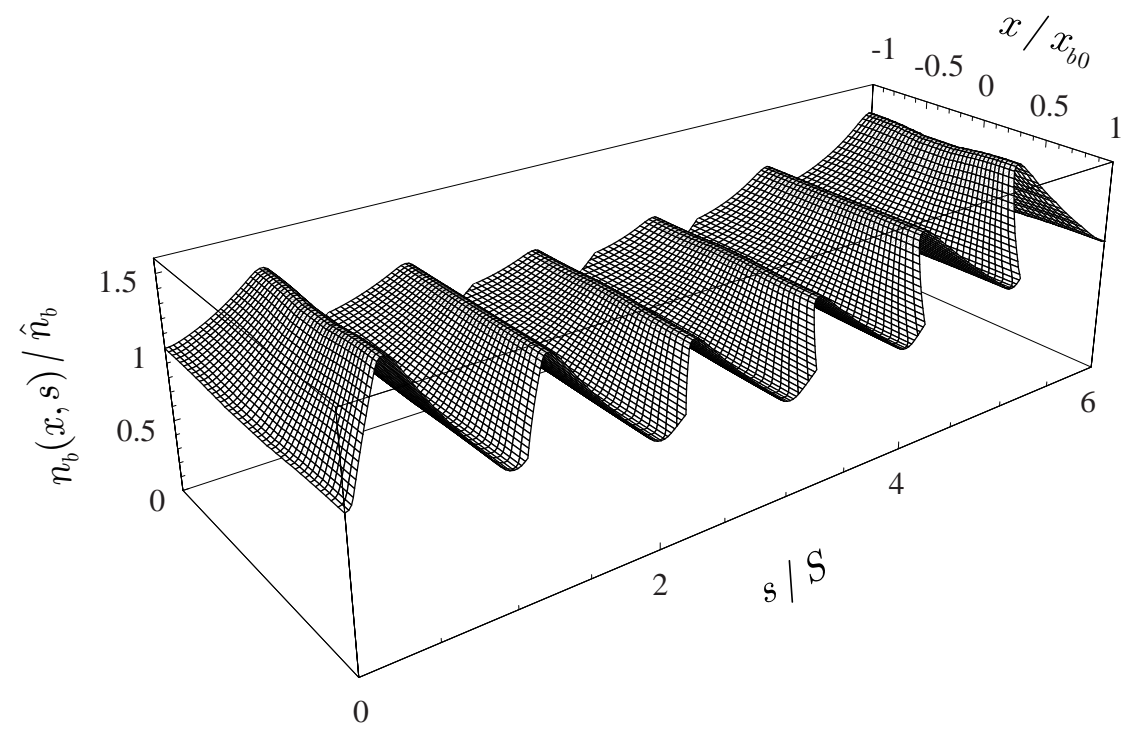

FIG. 8: Plot of laboratory-frame density profile $n_{b}(x, s)=n_{b}\left(x_{0}(x, s), s\right)$ obtained numerically from Eqs. (82) - (86) and Eq. (94) for the initial density profile in Eq. (90) and choice of system parameters in Eq. (93).

and

$$
V_{x b}\left(x_{0}, \tau\right)=-k_{\beta}\left[x_{0}-\frac{s_{b}}{\widehat{n}_{b}} \int_{0}^{x_{0}} d x_{0}^{\prime} n_{b}\left(x_{0}^{\prime}, 0\right)\right] \sin \left(k_{\beta} \tau\right),
$$

where we have introduced the notation $k_{\beta}=\sqrt{\kappa_{s f}}$. Furthermore, it follows from Eqs. 
(66) and (96) that the laboratory-frame variables $(x, s)$ and Lagrangian variables $\left(x_{0}, s\right)$ are related by

$$
\begin{aligned}
& x=x_{0}+\left[x_{0}-\frac{s_{b}}{\widehat{n}_{b}} \int_{0}^{x_{0}} d x_{0}^{\prime} n_{b}\left(x_{0}^{\prime}, 0\right)\right]\left[\cos \left(k_{\beta} \tau\right)-1\right], \\
& s=\tau .
\end{aligned}
$$

The condition for the back transformation (97) to remain single-valued is given by $\partial x / \partial x_{0}>$ 0 , which is equivalent to the requirement that the solution for $n_{b}\left(x_{0}, \tau\right)$ in Eq. (95) remain non-negative. Evaluating Eq. (95) or Eq. (97) at $k_{\beta} \tau=\pi$, some straightforward algebra that the condition for the transformation to remain single-valued is given by

$$
\frac{s_{b}}{\widehat{n}_{b}} n_{b}\left(x_{0}, 0\right)>\frac{1}{2},
$$

which assures that wave-breaking does not occur.

As a particular choice of initial density profile, we consider the case where

$$
n_{b}\left(x_{0}, 0\right)= \begin{cases}\widehat{n}_{b}\left[1+\Delta \cos \left(k_{0} x_{0}\right)\right], & 0 \leq|x|<x_{b 0}, \\ 0, & \left|x_{0}\right|>x_{b 0},\end{cases}
$$

which is illustrated in Fig. 9 for $k_{0} x_{b 0}=5 \pi / 2$ and $\Delta=0.45$. Note from Eq. (99) and Fig. 9 that $n_{b}\left(x_{0}, 0\right)$ corresponds to a sinusoidal density perturbation with amplitude $\Delta \widehat{n}_{b}$ superimposed on a flat-top density profile with constant density $\widehat{n}_{b}$. The corresponding transformation of variables consistent with Eqs. (97) and (99) is given by

$$
\begin{aligned}
k_{0} x & =k_{0} x_{0}+\left[\left(1-s_{b}\right) k_{0} x_{0}-s_{b} \Delta \sin \left(k_{0} x_{0}\right)\right]\left[\cos \left(k_{\beta} \tau\right)-1\right], \\
s & =\tau,
\end{aligned}
$$

and the inequality in Eq. (98) gives the requirement

$$
s_{b}(1-|\Delta|)>\frac{1}{2}
$$

which assures that the transformation in Eq. (100) remains single-valued. As a numerical example, we consider the choice of parameters

$$
s_{b}=1, \quad \Delta=0.45, \quad k_{0} x_{b 0}=\frac{5 \pi}{2} .
$$




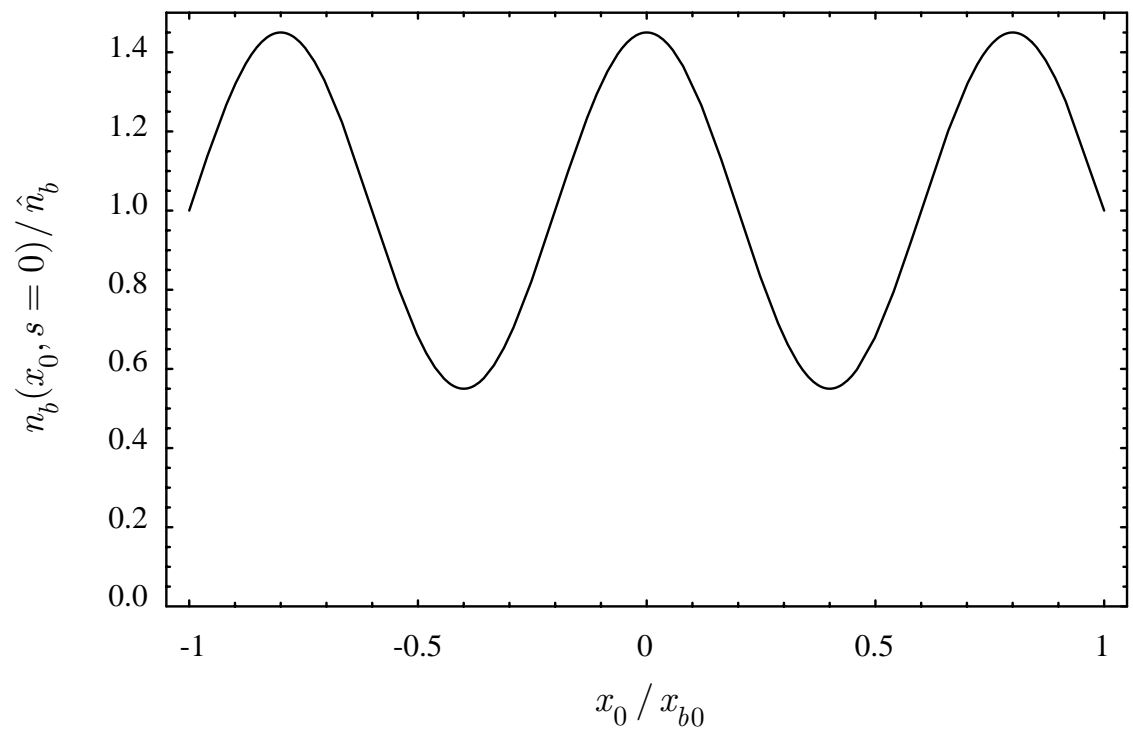

FIG. 9: Initial density profile in Eq. (99) for $k_{0} x_{b 0}=5 \pi / 2$ and $\Delta=0.45$.

Shown in Fig. 10 is a plot of the inverse transformation $x\left(x_{0}, s\right)$ versus $x_{0}$ obtained from Eq. (100) at $k_{\beta} s=0, \pi / 2, \pi, 3 \pi / 2,2 \pi$ for the choice of system parameters in Eq. (102), over the interval $k_{0}\left|x_{0}\right|<k_{0} x_{b 0}=5 \pi / 2$. Note from Fig. 10 that $\partial x / \partial x_{0}>0$ and the transformation remains single-valued, as expected. Consistent with Eqs. (95), (99) and (100), shown in Fig. 11 is a plot of the laboratory-frame density profile $n_{b}(x, s)=$ $n_{b}\left(x_{0}(x, s), \tau=s\right)$ obtained numerically for the choice of system parameters in Eq. (102). In Fig. 11, the density profile $n_{b}(x, s)$ is plotted over the beam cross-section $|x|<x_{b}(s)$. Of course, as a function of $s$, the density profile $n_{b}(x, s+L)=n_{b}(x, s)$ is periodic, with fundamental periodicity length $L=2 \pi / k_{\beta}$. What is most remarkable in Fig. 11 is that at $k_{\beta} s=\pi, 3 \pi, 5 \pi, \ldots$, very-large-amplitude density compression peaks with $\left[n_{b}\right]_{\max } / \widehat{n}_{b}=5.5$ occur at $k_{0} x= \pm 0.4$, and large-amplitude density rarefactions with $\left[n_{b}\right]_{\min } / \widehat{n}_{b}=0.55$ occur at $k_{0} x= \pm 0.4$. Therefore, in the context of the present cold-fluid model, the planar beam configuration supports large-amplitude collective oscillations, with peak density compression far exceeding the limiting space-charge density for a uniform density beam. (Keep in mind that $s_{b}=1$ has been assumed in the numerical example presented here.) 


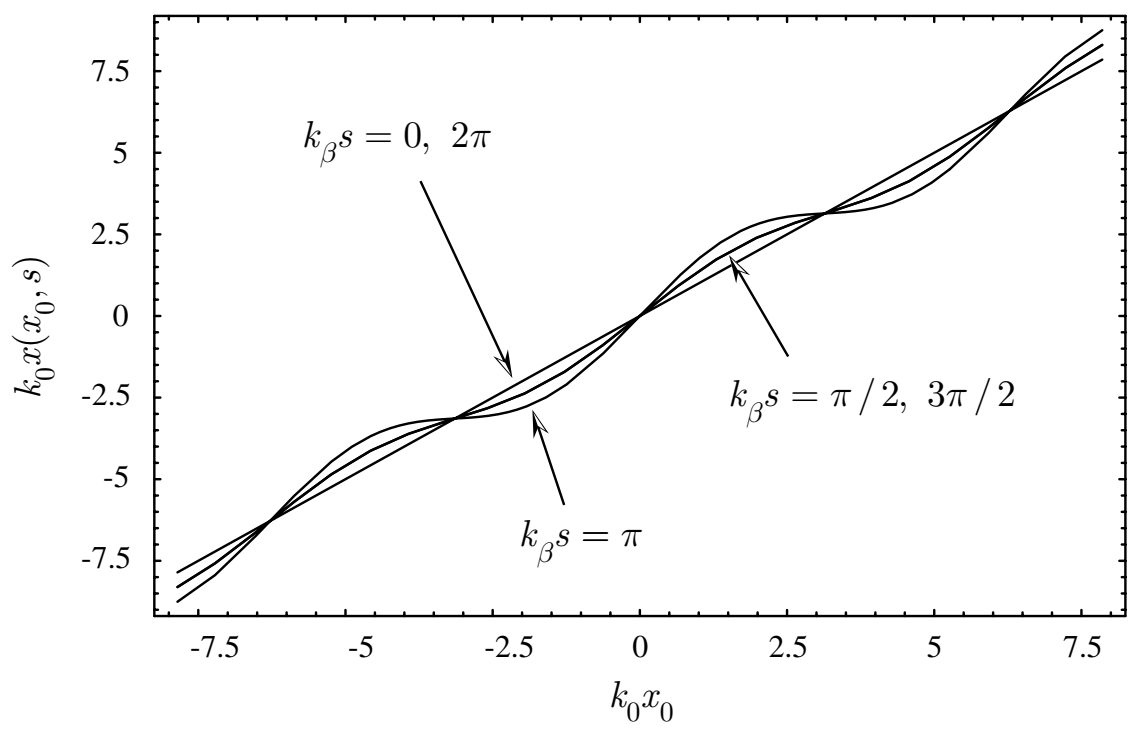

FIG. 10: Plot of $k_{0} x\left(x_{0}, s\right)$ versus $k_{0} x_{0}$ obtained from Eq. (100) at $k_{\beta} s=0, \pi / 2, \pi, 3 \pi / 2,2 \pi$ for the choice of system parameters $s_{b}=1, \Delta=0.45$ and $k_{0} x_{b 0}=5 \pi / 2$.

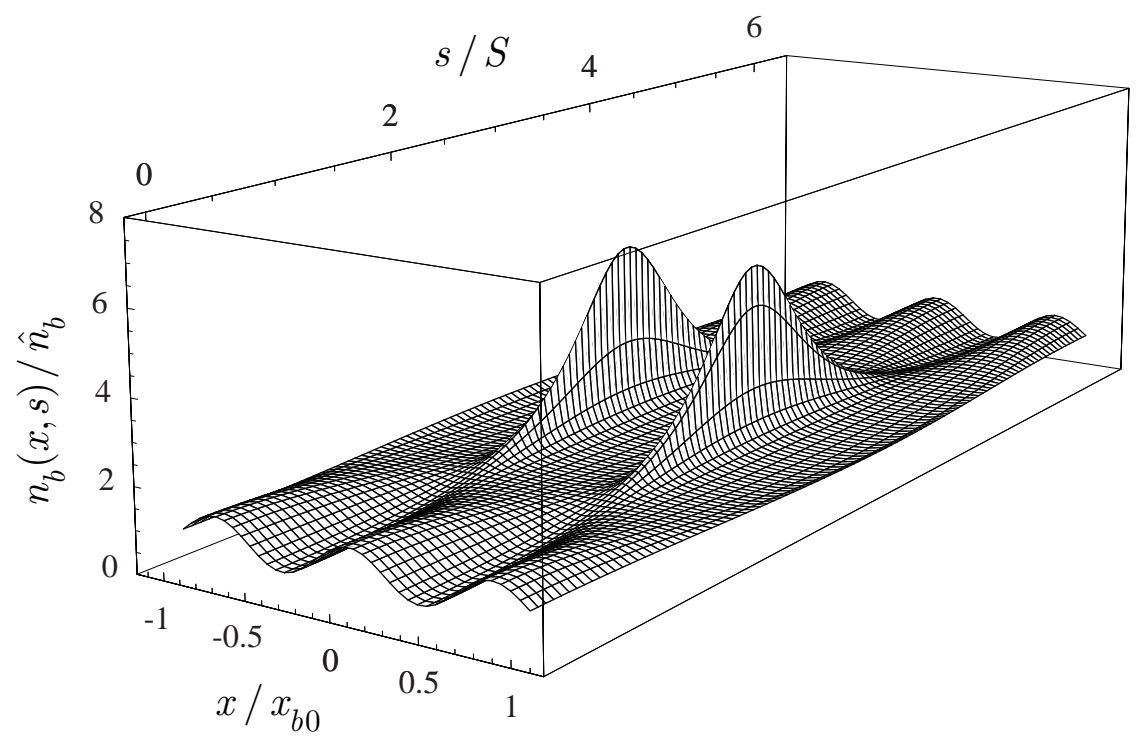

FIG. 11: Plot of laboratory-frame density profile $n_{b}(x, s)=n_{b}\left(x_{0}(x, s), \tau=s\right)$ obtained numerically from Eqs. (95), (99) and (100) for the choice of system parameters $s_{b}=1, \Delta=0.45$ and $k_{0} x_{b 0}=$ $5 \pi / 2$.

\section{CONCLUSIONS}

In this paper, the Vlasov-Maxwell equations (1) and (2) were used to investigate the evolution of an intense sheet beam with distribution function $f_{b}\left(x, x^{\prime}, s\right)$ propagating through 
a periodic focusing lattice $\kappa_{x}(s+S)=\kappa_{x}(s)$, where $S=$ const is the lattice period. The analysis considered the special class of distribution functions with uniform phase-space density $f_{b}\left(x, x^{\prime}, s\right)=A=$ const inside of the simply-connected boundary curves, $x_{+}^{\prime}(x, s)$ and $x_{-}^{\prime}(x, s)$, in the two-dimensional phase space $\left(x, x^{\prime}\right)$ [Eq. (23)]. Coupled nonlinear equations were derived describing the self-consistent evolution of the boundary curves, $x_{+}^{\prime}(x, s)$ and $x_{-}^{\prime}(x, s)$, and the self-field potential $\psi(x, s)=e_{b} \phi(x, s) / \gamma_{b} m_{b} \beta_{b}^{2} c^{2}$ [Eqs. (27) - (29)]. The resulting model was shown to be exactly equivalent to a (truncated) warm-fluid description with zero heat flow and triple-adiabatic equation-of-state with scalar pressure $P_{b}(x, s)=$ const $\times\left[n_{b}(x, s)\right]^{3}$ [Eqs. (36) - (38)]. Such a model is amenable to direct analysis by transforming to Lagrangian variables $\left(x_{0}, \tau\right)$ following the motion of a fluid element. This resulted in the single nonlinear partial differential equation (76) for the number density $n_{b}\left(x_{0}, \tau\right)$ of beam particles in Lagrangian variables, with back transformation to the laboratory-frame variables $(x, s)$ specified by Eq. (66). Specific examples of periodicallyfocused beam equilibria were presented, ranging from a finite-emittance beam in which the boundary curves in phase space correspond to a pulsating parallelogram (Sec. IV), to a cold beam in which the number density of beam particles exhibits large-amplitude periodic oscillations (Sec. V). For the case of a sheet beam with uniform phase-space density [Eq. (23)], the present analysis clearly demonstrates the existence of periodically focused beam equilibria without the undesirable feature of an inverted population in phase space that is characteristic of a KV beam distribution. In future work, the warm-fluid model developed in Secs. III.B and V.I will be used to derive a nonlinear Schroedinger equation describing the evolution of perturbations about a uniform density beam, including soliton solutions. It should be emphasized that the existence of periodically-focused beam equilibrium for a non-KV distribution with uniform density in the two-dimensional phase space $\left(x, x^{\prime}\right)$ does not imply that periodically focused beam equilibria exist for non-KV beam distributions in four- and six-dimensions. 


\section{Acknowledgments}

This research was supported by the Department of Energy under contract AC02$76 \mathrm{CH} 03073$.

[1] R.C. Davidson and H. Qin, Physics of Intense Charged Particle Beams in High Energy Accelerators (World Scientific, Singapore, 2001), and references therein.

[2] M. Reiser, Theory and Design of Charged Particle Beams (John Wiley \& Sons, Inc., New York, 1994).

[3] A.W. Chao, Physics of Collective Beam Instabilities in High Energy Accelerators (John Wiley \& Sons, Inc., New York, 1993).

[4] See, for example, Proceedings of the 2001 Particle Accelerator Conference (IEEE Catalog Number 01CH37268), pp. 1-4098.

[5] See, for example, Proceedings of the 1999 International Heavy Ion Fusion Symposium, Nuclear Instruments and Methods in Physics Research A464, pp. 1-674 (2001).

[6] I.M. Kapchinskij and V.V Vladimirskij, in Proceedings of the International Conference on High Energy Accelerators and Instrumentation (CERN Scientific Information Service, Geneva, 1959), p. 274.

[7] R.L. Gluckstern, in Proceedings of the 1970 Proton Linear Accelerator Conference, Batavia, IL, edited by M.R. Tracy (National Accelerator Laboratory, Batavia, IL, 1971), p. 811.

[8] T.-S. Wang and L. Smith, Particle Accelerators 12, 247 (1982).

[9] I. Hofmann, L.J. Laslett, L. Smith and I. Haber, Particle Accelerators 13, 145 (1983).

[10] J. Struckmeier, J. Klabunde and M. Reiser, Particle Accelerators 15, 47 (1984).

[11] I. Hofmann and J. Struckmeier, Particle Accelerators 21, 69 (1987).

[12] J. Struckmeier and I. Hofmann, Particle Accelerators 39, 219 (1992).

[13] N. Brown and M. Reiser, Physics of Plasmas 2, 965 (1995).

[14] R.C. Davidson and H. Qin, Physical Review Special Topics on Accelerators and Beams 2, $114401(1999)$.

[15] R.L. Gluckstern, W.-H. Cheng and H. Ye, Physical Review Letters 75, 2835 (1995).

[16] R.C. Davidson and C. Chen, Particle Accelerators 59, 175 (1998). 
[17] C. Chen, R. Pakter and R.C. Davidson, Physical Review Letters 79, 225 (1997).

[18] C. Chen and R.C. Davidson, Physical Review E49, 5679 (1994).

[19] R.C. Davidson, W.W. Lee and P. Stoltz, Physics of Plasmas 5, 279 (1998).

[20] R.C. Davidson, Physical Review Letters 81, 991 (1998).

[21] R.C. Davidson, Physics of Plasmas 5, 3459 (1998).

[22] H. Qin, R.C. Davidson and W.W. Lee, Physical Review Special Topics on Accelerators and Beams 3, 084401 (2000); 3, 109901 (2000).

[23] P. Stoltz, R.C. Davidson and W.W. Lee, Physics of Plasmas 6, 298 (1999).

[24] W.W. Lee, Q. Qian and R.C. Davidson, Physics Letters A230, 347 (1997).

[25] Q. Qian, W.W. Lee and R.C. Davidson, Physics of Plasmas 4, 1915 (1997).

[26] A. Friedman, D.P. Grote and I. Haber, Physics of Fluids B4, 2203 (1992).

[27] S.M. Lund, J.J. Barnard, G.D. Craig, A. Friedman, D.P. Grote, T.S. Sangster, W.M. Sharp, S. Eylon, T.T. Fessenden, E. Henestroza, S. Yu and I. Haber, Nuclear Instruments and Methods in Physics Research A415, 345 (1998).

[28] A. Friedman, J.J. Barnard, D.P. Grote and I. Haber, Nuclear Instruments and Methods in Physics Research A415, 455 (1998).

[29] I. Haber, A. Friedman, D.P. Grote, S.M. Lund and R.A. Kishek, Physics of Plasmas 6, 2254 (1999).

[30] R.A. Kishek, P.G. O'Shea and M. Reiser, Physical Review Letters 85, 4514 (2000).

[31] S.I. Tzenov and R.C. Davidson, Physical Review Special Topics on Accelerators and Beams 5, $021001(2002)$.

[32] R.C. Davidson and H. Qin, Physical Review Special Topics on Accelerators and Beams 4, 104401 (2001).

[33] R.C. Davidson, H. Qin and P.J. Channell, Physical Review Special Topics on Accelerators and Beams 2, 074401 (1999); 3, 029901 (2000).

[34] P.J. Channell, Physics of Plasmas 6, 982 (1999).

[35] R.C. Davidson, Methods in Nonlinear Plasma Theory (Academic Press, New York, 1972), Chapter 2 and references therein.

[36] L.D. Landau and E.M. Lifshitz, Fluid Mechanics (Pergamon Press, Oxford, 1987).

[37] See, for example, Chapters $5-8$ of Ref. 1.

[38] See, for example, Chapter 6 of Ref. 1. 


\section{External Distribution}

Plasma Research Laboratory, Australian National University, Australia

Professor I.R. Jones, Flinders University, Australia

Professor João Canalle, Instituto de Fisica DEQ/IF - UERJ, Brazil

Mr. Gerson O. Ludwig, Instituto Nacional de Pesquisas, Brazil

Dr. P.H. Sakanaka, Instituto Fisica, Brazil

The Librarian, Culham Laboratory, England

Mrs. S.A. Hutchinson, JET Library, England

Professor M.N. Bussac, Ecole Polytechnique, France

Librarian, Max-Planck-Institut für Plasmaphysik, Germany

Jolan Moldvai, Reports Library, MTA KFKI-ATKI, Hungary

Dr. P. Kaw, Institute for Plasma Research, India

Ms. P.J. Pathak, Librarian, Insitute for Plasma Research, India

Ms. Clelia De Palo, Associazione EURATOM-ENEA, Italy

Dr. G. Grosso, Instituto di Fisica del Plasma, Italy

Librarian, Naka Fusion Research Establishment, JAERI, Japan

Library, Plasma Physics Laboratory, Kyoto University, Japan

Research Information Center, National Institute for Fusion Science, Japan

Dr. O. Mitarai, Kyushu Tokai University, Japan

Dr. Jiangang Li, Institute of Plasma Physics, Chinese Academy of Sciences, People's Republic of China

Professor Yuping Huo, School of Physical Science and Technology, People's Republic of China

Library, Academia Sinica, Institute of Plasma Physics, People's Republic of China

Librarian, Institute of Physics, Chinese Academy of Sciences, People's Republic of China

Dr. S. Mirnov, TRINITI, Troitsk, Russian Federation, Russia

Dr. V.S. Strelkov, Kurchatov Institute, Russian Federation, Russia

Professor Peter Lukac, Katedra Fyziky Plazmy MFF UK, Mlynska dolina F-2, Komenskeho Univerzita, SK-842 15 Bratislava, Slovakia

Dr. G.S. Lee, Korea Basic Science Institute, South Korea

Institute for Plasma Research, University of Maryland, USA

Librarian, Fusion Energy Division, Oak Ridge National Laboratory, USA

Librarian, Institute of Fusion Studies, University of Texas, USA

Librarian, Magnetic Fusion Program, Lawrence Livermore National Laboratory, USA

Library, General Atomics, USA

Plasma Physics Group, Fusion Energy Research Program, University of California at San Diego, USA

Plasma Physics Library, Columbia University, USA

Alkesh Punjabi, Center for Fusion Research and Training, Hampton University, USA

Dr. W.M. Stacey, Fusion Research Center, Georgia Institute of Technology, USA

Dr. John Willis, U.S. Department of Energy, Office of Fusion Energy Sciences, USA

Mr. Paul H. Wright, Indianapolis, Indiana, USA 
The Princeton Plasma Physics Laboratory is operated by Princeton University under contract with the U.S. Department of Energy.

\author{
Information Services \\ Princeton Plasma Physics Laboratory \\ P.O. Box 451 \\ Princeton, NJ 08543
}

Phone: 609-243-2750

Fax: 609-243-2751

e-mail: pppl_info@pppl.gov

Internet Address: http://www.pppl.gov 